\title{
The homotopy theory of Khovanov homology
}

\author{
BRENT EVERITT \\ PAUl TURNER
}

\begin{abstract}
We show that the unnormalised Khovanov homology of an oriented link can be identified with the derived functors of the inverse limit. This leads to a homotopy theoretic interpretation of Khovanov homology.
\end{abstract}

57M27; 55P42

\section{Motivation and introduction}

In order to apply the methods of homotopy theory to Khovanov homology there are several natural approaches. One is to build a space or spectrum whose classical invariants give Khovanov homology, then show its homotopy type is a link invariant, and finally study this space using homotopy theory. Ideally this approach would begin with some interesting geometry and lead naturally to Khovanov homology. One also might hope to construct something more refined than Khovanov homology in this way (see Lipshitz and Sarkar [12] for a combinatorial approach to this). Another approach is to interpret the existing constructions of Khovanov homology in homotopy theoretic terms. By placing the constructions into a homotopy setting one makes Khovanov homology amenable to the methods and techniques of homotopy theory. In this paper our interest is with the second of these approaches. Our aim is to show that Khovanov homology can be interpreted in a homotopy theoretic way using homotopy limits and to subsequently develop a number of results about the specific type of homotopy limit arising. The latter will provide homotopy tools appropriate for studying Khovanov homology.

Recall that the central combinatorial input for Khovanov homology is the decorated "cube" of resolutions based on a link diagram $D$ (see Section 1.1). As we explain later, it is convenient to view this cube as a presheaf of abelian groups over a certain poset $\boldsymbol{Q}$, that is, as a functor $F_{K H}: \boldsymbol{Q}^{\text {op }} \rightarrow \mathbf{A b}$.

In the first section we show that Khovanov homology can be described in terms of the right derived functors of the inverse limit of this presheaf. 
Theorem 1.4 Let $D$ be a link diagram and let $F_{K H}: Q^{\text {op }} \rightarrow \mathbf{A b}$ be the Khovanov presheaf defined in Section 1.1. Then

$$
\overline{K H}^{i}(D) \cong{\overleftarrow{Q^{\circ \mathrm{p}}}}^{i} F_{K H}
$$

On the left we have singly graded unnormalised Khovanov homology (see Section 1.1) while on the right we have the $i^{\text {th }}$ derived functor of the inverse limit (see Section 1.2). This result is central to the homotopy theoretic interpretation of Khovanov homology but is also of independent interest: many cohomology theories are defined as the right derived functors of some interesting partially exact functor, or at least can be described in such terms. Examples include group cohomology, sheaf cohomology and Hochschild cohomology. Obtaining a description in these terms for Khovanov homology reveals its similarity to existing theories, not apparent from the original definition. Moreover it opens up Khovanov homology to the many techniques available to cohomology theories defined as right derived functors. Also the construction given in this paper is functorial with respect to morphisms of presheaves, which, being more general, may offer calculational advantage. By connecting with a more familiar description of higher derived functors we also obtain a description of Khovanov homology as the cohomology of the classifying space equipped with a system of local coefficients as described in Proposition 1.6.

Right derived functors of a presheaf of abelian groups can be interpreted in homotopy theoretic terms by way of the homotopy limit of the corresponding diagram of EilenbergMac Lane spaces. In the second section we recall basic facts about homotopy limits before returning to Khovanov homology. We compose the Eilenberg-Mac Lane space functor $K(-, n)$ with the Khovanov presheaf $F_{K H}$ of a link diagram to obtain a diagram of spaces $\mathscr{F}_{n}: \boldsymbol{Q}^{\text {op }} \rightarrow$ Sp whose homotopy limit $\boldsymbol{Y}_{n} D=\operatorname{holim}_{\boldsymbol{Q}^{\text {op }}} \mathscr{F}_{n}$ has homotopy groups described in the following proposition.

\section{Proposition 2.8}

$$
\pi_{i}\left(\boldsymbol{Y}_{n} D\right) \cong \begin{cases}\overline{K H}^{n-i}(D) & \text { if } 0 \leq i \leq n \\ 0 & \text { else. }\end{cases}
$$

For rather elementary reasons the space $Y_{n} D$ is seen to be a product of Eilenberg-Mac Lane spaces and thus determined by the Khovanov homology. Thus the problem of defining an invariant space or spectrum (a homotopy type) is "solved" by the above as well, but in an uninteresting way. Nevertheless we now find ourselves within a homotopy theory context so we can apply its methods and techniques to Khovanov homology.

In the third section we develop this perspective further by isolating a result about holim and homotopy fibres in this specific situation which may be useful in the study 
of Khovanov homology. One central point is that in the presheaf setting (or using chain complexes) one has long exact sequences in homology arising from short exact sequences of presheaves. Typically the latter arise from a given injection or surjection and one requires some luck for this to be the case. In the homotopy setting, by contrast, any map of spaces has a homotopy fibre and an attendant long exact sequence in homotopy groups. We illustrate the use of this calculus in the last section where we discuss the skein relation as the homotopy long exact sequence of the smoothing change map, reprove Reidemeister invariance from the homotopy perspective and make an explicit computation.

We have tried as far as possible to make this article readable both by knot theorists interested in Khovanov homology and by homotopy theorists with a passing interest in knot theory.

\section{Acknowledgements}

We thank Hans-Werner Henn, Kathryn Hess, Robert Lipshitz, Sucharit Sarkar, Jérôme Scherer and the referee for helpful remarks.

\section{Khovanov homology and higher inverse limits}

The main result of this section is a reinterpretation of the (unnormalised) Khovanov homology of a link as the derived functors of $\lim _{\longleftarrow}$ over a certain small category.

\subsection{A modified Boolean lattice and the inverse limit}

Let $\mathbf{B}=\mathbf{B}_{A}$ be the Boolean lattice on a set $A$ : the poset of subsets of $A$ ordered by reverse inclusion. We write $\leq$ for the partial order and $\prec$ for the covering relation, ie given subsets $x$ and $y$, then $x \leq y$ when $x \supseteq y$ and $x \prec y$ when $x$ is obtained from $y$ by adding a single element.

Now let $D$ be a link diagram and $\mathbf{B}$ the Boolean lattice on the set of crossings of $D$. Each crossing of $D$ can be 0 - or 1-resolved

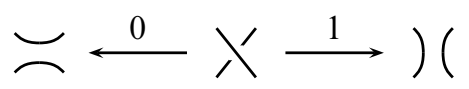

and if $x$ is some subset of the crossings, then the complete resolution $D(x)$ is what results from 1 -resolving the crossings in $x$ and 0 -resolving the crossings not in $x$. It is a collection of planar circles. 
Let $V=\mathbb{Z}[1, u]$ where $\mathbb{Z}[S]$ is the free abelian group on the set $S$. This rank two abelian group becomes a Frobenius algebra using the maps $m: V \otimes V \rightarrow V, \epsilon: V \rightarrow \mathbb{Z}$ and $\Delta: V \rightarrow V \otimes V$ defined by

$$
\begin{aligned}
m: & 1 \otimes 1 \mapsto 1, \quad 1 \otimes u \mapsto u, \quad u \otimes 1 \mapsto u, \quad u \otimes u \mapsto 0 \\
\epsilon: & 1 \mapsto 0, \quad u \mapsto 1 ; \\
\Delta: & 1 \mapsto 1 \otimes u+u \otimes 1, \quad u \mapsto u \otimes u .
\end{aligned}
$$

The "Khovanov cube" is obtained by assigning abelian groups to the elements of $\mathbf{B}$ and homomorphisms between the groups associated to comparable elements. One says "cube" as the Hasse diagram of the poset $\mathbf{B}_{A}$ is the $|A|$-dimensional cube, with edges given by the covering relations.

For $x \in \mathbf{B}$ let $F_{K H}(x)=V^{\otimes k}$, with a tensor factor corresponding to each connected component of $D(x)$. If $x \prec y$ in $\mathbf{B}$ then $D(x)$ results from 1 -resolving a crossing that was 0 -resolved in $D(y)$, with the qualitative effect that two of the circles in $D(y)$ fuse into one in $D(x)$, or one of the circles in $D(y)$ bifurcates into two in $D(x)$. In the first case $F_{K H}(x \prec y): F_{K H}(y)=V^{\otimes k} \rightarrow V^{\otimes k-1}=F_{K H}(x)$ is the map using $m$ on the tensor factors corresponding to the fused circles, and the identity on the others. In the second, $F_{K H}(x \prec y): F_{K H}(y)=V^{\otimes k} \rightarrow V^{\otimes k+1}=F_{K H}(x)$ is the map using $\Delta$ on the tensor factor corresponding to the bifurcating circles, and the identity on the others.

All of this is most concisely expressed by regarding $\mathbf{B}$ as a category with objects the elements of $\mathbf{B}$ and with a unique morphism $x \rightarrow y$ whenever $x \leq y$. The decoration by abelian groups is then nothing other than a covariant functor, or presheaf,

$$
F_{K H}: \mathbf{B}^{\text {op }} \rightarrow \mathbf{A b},
$$

where $\mathbf{A b}$ is the category of abelian groups. The diagram $D$ is suppressed from the notation.

Each square face of the cube $\mathbf{B}$ is sent by the functor $F_{K H}$ to a commutative diagram of abelian groups. To extract a cochain complex from the decorated cube these squares must anticommute, and this is achieved by adding \pm signs to the edges of the cube so that each square face has an odd number of - signs on its edges. We write $[x, y]$ for the sign associated to the edge $x \prec y$ of $\mathbf{B}$. The Khovanov complex $K^{*}$ has $n$-cochains $K^{n}=\bigoplus_{|x|=n} F_{K H}(x)$, the direct sum over the subsets of size $n$ (or rows of the cube), and differential $d: K^{n-1} \rightarrow K^{n}$ given by $d=\sum[x, y] F_{K H}(x \prec y)$, the sum over all pairs $x \prec y$ with $x$ of size $n$ (or sum of all signed maps between rows $n-1$ and $n$ ). That $d$ is a differential follows immediately from the anticommutativity of the signage. 
Definition 1.1 The unnormalised Khovanov homology of a link diagram $D$ is defined as the homology of the Khovanov cochain complex:

$$
\overline{K H}^{*}(D)=H\left(K^{*}, d\right)
$$

The normalised Khovanov homology of an oriented link diagram $D$ with $c$ negative crossings is a shifted version of the above:

$$
K H^{*}(D)=\overline{K H}^{*+c}(D)
$$

The normalised Khovanov homology is a link invariant. All of the above is standard and there are several reviews of this material available (see for example Bar-Natan [1], Turner [17] and Khovanov [11]).

A note on the $\boldsymbol{q}$-grading Usually there is an internal grading on Khovanov homology making it a bigraded theory. This " $q$-grading" is important in recovering the Jones polynomial. A huge amount of information is retained however even if this grading is completely ignored. For example Khovanov homology detects the unknot with or without the $q$-grading. In this paper the $q$-grading plays no role and we consider the Frobenius algebra $V$ above as ungraded, resulting in a singly graded theory.

For what follows we need to modify the poset $\mathbf{B}$ in a seemingly innocuous way, but one which has considerable consequences (see also the remarks at the end of Section 1.3). There is a unique maximal element $\mathbf{1} \in \mathbf{B}$ (corresponding to the empty subset of $A$ ) with $x \leq \mathbf{1}$ for all $x \in \mathbf{B}$. Now formally adjoin to $\mathbf{B}$ an additional maximal element $\mathbf{1}^{\prime}$ such that $x \leq \mathbf{1}^{\prime}$ for all $x \in \mathbf{B}$ with $x \neq \mathbf{1}$, and denote the resulting poset (category) by $\boldsymbol{Q}=\boldsymbol{Q}_{A}$. Extend $F_{K H}$ to a (covariant) functor

$$
F_{K H}: Q^{\text {op }} \rightarrow \mathbf{A b}
$$

by setting $F_{K H}\left(\mathbf{1}^{\prime}\right)=0$ and $F_{K H}\left(x \rightarrow \mathbf{1}^{\prime}\right): F_{K H}\left(\mathbf{1}^{\prime}\right) \rightarrow F_{K H}(x)$ to be the only possible homomorphism.

The construction of $K^{*}$ extends verbatim to $Q$ : the chains are the direct sum over the rows of $\boldsymbol{Q}$ (identical to $\mathbf{B}$ except for the top row where the zero group is added) and the differential is the sum of signed maps between consecutive rows-again identical except between the first and second rows; we adopt the convention $\left[x, \mathbf{1}^{\prime}\right]=-1$ for an $x$ with $x \prec \mathbf{1}^{\prime}$. The resulting homology is easily seen to be the unnormalised Khovanov homology again.

It will be convenient later to identify $\boldsymbol{Q}$ with the poset of cells of a certain CW complex. Recall that a CW complex $X$ is regular if for any cell $x$ the characteristic map $\Phi_{x}:\left(B^{k}, S^{k-1}\right) \rightarrow\left(X^{k-1} \cup x, X^{k-1}\right)$ is a homeomorphism of $B^{k}$ onto its image. 

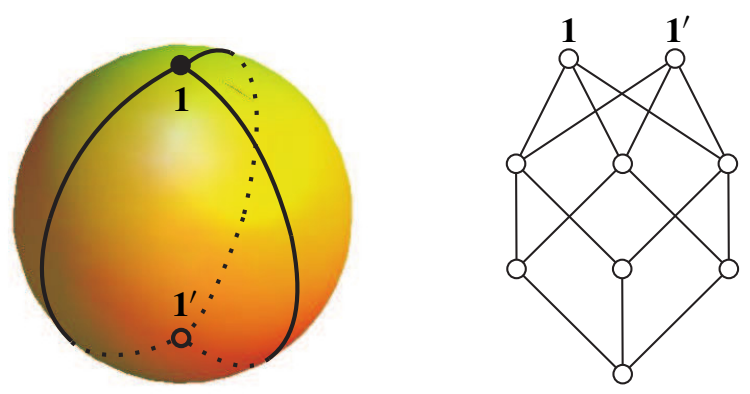

Figure 1: Regular CW complex $X$ (left) with cell poset $\boldsymbol{Q}_{A}$ (right) for $|A|=3$

We can then define a partial order on the cells of $X$ by $x \leq y$ exactly when $\bar{x} \supseteq y$, where $\bar{x}$ is the (CW) closure of the cell.

To realise $\boldsymbol{Q}_{A}$ as such a thing suppose that $|A|=n$ and let $\Delta^{n-1}$ be an $(n-1)-$ simplex. Let $X$ be the suspension $S \Delta^{n-1}$, an $n$-ball, and take the obvious CW decomposition of $X$ with two 0 -cells (the suspension points) and all other cells the suspensions $S x$ of the cells $x$ of $\Delta^{n-1}$. As the suspension of cells preserves the inclusions $\bar{x} \supseteq y$ and the two 0 -cells are maximal with respect to this, we get that $X$ has cell poset $\boldsymbol{Q}$. An $x \in \boldsymbol{Q}$ corresponds to an $|x|$-dimensional cell of $X$; the case $n=3$ is depicted in Figure 1.

Using the signage introduced above, if $x$ is a 1 -cell we have $[x, \mathbf{1}]+\left[x, \mathbf{1}^{\prime}\right]=0$; if $\operatorname{dim} x-\operatorname{dim} y=2$ and $z_{1}, z_{2}$ are the unique cells with $x \prec z_{i} \prec y$, then $\left[x, z_{1}\right]\left[z_{1}, y\right]+$ $\left[x, z_{2}\right]\left[z_{2}, y\right]=0$. These properties then ensure that there are orientations for the cells of $X$ so that $[x, y]$ is the incidence number of the cells $x$ and $y$ (see Massey [13, Chapter IX, Theorem 7.2]).

We finish this introductory subsection by recalling the definition of the inverse limit of abelian groups. Let $\mathbf{C}$ be a small category and $F: \mathbf{C} \rightarrow \mathbf{A b}$ a functor. Then the inverse limit $\lim _{\mathbf{C}} F$ is an abelian group that is universal with respect to the property that for all $x \in \mathbf{C}$ there are homomorphisms $\lim _{\mathbf{C}} F \rightarrow F(x)$ that commute with the homomorphisms $F(x) \rightarrow F\left(x^{\prime}\right)$ for all morphisms $x \rightarrow x^{\prime}$ in $\mathbf{C}$. The limit is constructed by taking the subgroup of the product $\prod_{x \in \mathbf{C}} F(x)$ consisting of those $\mathbf{C}$-tuples $\left(\alpha_{x}\right)_{x \in \mathbf{C}}$ such that for all morphisms $x \rightarrow x^{\prime}$, the induced map $F(x) \rightarrow F\left(x^{\prime}\right)$ sends $\alpha_{x}$ to $\alpha_{x^{\prime}}$.

It is an easy exercise to see that $\lim _{Q^{\text {op }}} F_{K H}=\operatorname{ker} d^{0}$, the degree zero differential of the cochain complex $K^{*}$, and so

$$
{\underset{\lim }{\text { op }}} F_{K H} \cong \overline{K H}^{0}(D)
$$




\subsection{Derived functors of the inverse limit}

We have seen that presheaves of abelian groups provide a convenient language for the construction of Khovanov homology, and that the inverse limit of the presheaf $F_{K H}: \boldsymbol{Q}^{\mathrm{op}} \rightarrow \mathbf{A b}$ captures this homology in degree zero. In this subsection we review general facts about the category of presheaves, the inverse limit functor and its derived functors. These "higher limits" give, by definition, the cohomology of a small category $\mathbf{C}$ with coefficients in a presheaf. The moral is that they are computed using projective resolutions for the trivial (or constant) presheaf, just as group cohomology, say, is computed using projective resolutions for the trivial $G$-module. The material here is standard (see eg Weibel [19, Chapter 2]) and obviously holds in greater generality; rather than working in the category ${ }_{R}$ Mod of modules over a commutative ring $R$, we content ourselves with $\mathbf{A b}:={ }_{\mathbb{Z}}$ Mod. In the following subsection we will show that the higher limits capture Khovanov homology in all degrees, not just degree zero.

Recall that a presheaf on a small category $\mathbf{C}$ is a (covariant) functor $F: \mathbf{C}^{\mathrm{op}} \rightarrow \mathbf{A b}$. The category $\operatorname{PreSh}(\mathbf{C})=\mathbf{A b}^{\mathbf{C}^{\text {op }}}$ has as objects the presheaves $F: \mathbf{C}^{\text {op }} \rightarrow \mathbf{A b}$ and as morphisms the natural transformations $\tau: F \rightarrow G$. For $x \in \mathbf{C}$, we write $F(x)$ for its image in $\mathbf{A b}$ and $\tau_{x}$ for the map $F(x) \rightarrow G(x)$ making up the component at $x$ of the natural transformation $\tau$.

$\operatorname{PreSh}(\mathbf{C})$ is an abelian category having enough projective and injective objects. Many basic constructions in PreSh(C), such as kernels, cokernels, decisions about exactness, etc, can be constructed locally, or "pointwise", eg the value of the presheaf $\operatorname{ker}(\tau: F \rightarrow G)$ at $x \in \mathbf{C}$ is $\operatorname{ker}\left(\tau_{x}: F(x) \rightarrow G(x)\right)$, and similarly for images. In particular, a sequence of presheaves $F \rightarrow G \rightarrow H$ is exact if and only if for all $x \in \mathbf{C}$ the local sequence $F(x) \rightarrow G(x) \rightarrow H(x)$ is exact.

The simplest presheaf is the constant one: if $A \in \mathbf{A b}$, define $\Delta A: \mathbf{C}^{\mathrm{op}} \rightarrow \mathbf{A b}$ by $\Delta A(x)=A$ for all $x$, and for all morphisms $x \rightarrow y$ in $\mathbf{C}$ let $\Delta A(x \rightarrow y)=$ 1: $\Delta A(y) \rightarrow \Delta A(x)$. If $f: A \rightarrow B$ is a map of abelian groups then there is a natural transformation $\tau: \Delta A \rightarrow \Delta B$ with $\tau_{x}: \Delta A(x) \rightarrow \Delta B(x)$ the map $f$. Thus we have the constant sheaf functor $\Delta: \mathbf{A b} \rightarrow \operatorname{PreSh}(\mathbf{C})$ which is easily seen to be exact.

We saw at the end of Section 1.1 that the inverse $\operatorname{limit} \lim F$ exists in $\mathbf{A b}$ for any presheaf $F \in \operatorname{PreSh}(\mathbf{C})$. Indeed, we have a (covariant) functor $\underset{\leftarrow}{\lim } \mathbf{P} \operatorname{PreSh}(\mathbf{C}) \rightarrow \mathbf{A b}$ by universality. For any $A \in \mathbf{A b}$ and any $F \in \operatorname{PreSh}(\mathbf{C})$ there are natural bijections

$$
\operatorname{Hom}_{\mathbf{P r e S h}(\mathbf{C})}(\Delta A, F) \cong \operatorname{Hom}_{\mathbb{Z}}\left(A, \lim _{\longleftarrow} F\right)
$$

so that $\lim _{\leftarrow}$ is right adjoint to $\Delta$. In particular $\lim _{\longleftarrow}$ is left exact, and we have the right derived $\overleftarrow{\text { functors }}$

$$
\lim ^{i}:=R^{i} \underset{\leftarrow}{\lim } \operatorname{PreSh}(\mathbf{C}) \rightarrow \mathbf{A b} \quad(i \geq 0)
$$


with $\lim ^{0}$ naturally isomorphic to $\underset{\leftarrow}{\lim }$.

A special case of the adjointness (2) is the following: For any presheaf $F$ over $\mathbf{C}$ the universality of the limit gives a homomorphism $\operatorname{Hom}_{\operatorname{PreSh}(\mathbf{C})}(\Delta \mathbb{Z}, F) \rightarrow \underset{\longleftarrow}{\lim } F$ that sends a natural transformation $\tau \in \operatorname{Hom}_{\operatorname{PreSh}(\mathbf{C})}(\Delta \mathbb{Z}, F)$ to the tuple $\left(\tau_{x}(1)\right)_{x \in \mathbf{C}}^{\longleftarrow} \underset{\leftarrow}{\lim } F$. This is in fact a natural isomorphism, so we have a natural isomorphism of functors

$$
\lim \cong \operatorname{Hom}_{\operatorname{PreSh}(\mathbf{C})}(\Delta \mathbb{Z},-)
$$

and thus

$$
\lim ^{i} \cong R^{i} \operatorname{Hom}_{\operatorname{PreSh}(\mathbf{C})}(\Delta \mathbb{Z},-) \text { for all } i \geq 0
$$

If $0 \rightarrow F \rightarrow G \rightarrow H \rightarrow 0$ is a short exact sequence in $\operatorname{PreSh}(\mathbf{C})$ then there is a long exact sequence in $\mathbf{A b}$ :

$$
0 \longrightarrow \lim F \longrightarrow \lim _{\longleftarrow}^{\lim } \longrightarrow \lim H \longrightarrow \cdots \lim ^{i} F \longrightarrow \lim ^{i} G \longrightarrow \lim ^{i} H \longrightarrow
$$

It turns out that the derived functors of the covariant Hom functor in (3) can be replaced by the derived functors of the contravariant Hom functor. Let $F, G$ be presheaves over the small category $\mathbf{C}$. Then

$$
R^{i} \operatorname{Hom}_{\operatorname{PreSh}(C)}(F,-)(G) \cong R^{i} \operatorname{Hom} \operatorname{PreSh}(C)(-, G)(F)
$$

for all $i \geq 0$. One thinks of this as a "balancing Ext" result for presheaves. The corresponding result in ${ }_{R}$ Mod is [19, Theorem 2.7.6], and the reader can check that the proof given there goes straight through in $\operatorname{PreSh}(C)$. Summarising, for $F \in \operatorname{PreSh}(C)$,

$$
\lim ^{i}(F) \cong\left(R^{i} \operatorname{Hom}_{\operatorname{PreSh}(\mathbf{C})}(\Delta \mathbb{Z},-)\right)(F) \cong\left(R^{i} \operatorname{Hom}_{\mathbf{P r e S h}(\mathbf{C})}(-, F)\right)(\Delta \mathbb{Z}) .
$$

To compute the right derived functors of a contravariant functor like $\operatorname{Hom}_{\operatorname{PreSh}(\mathbf{C})}(-, F)$, we use a projective resolution. Let $P_{*} \rightarrow \Delta \mathbb{Z}$ be a projective resolution for $\Delta \mathbb{Z}$, ie an exact sequence

$$
\cdots \stackrel{\delta}{\rightarrow} P_{2} \stackrel{\delta}{\rightarrow} P_{1} \stackrel{\delta}{\rightarrow} P_{0} \stackrel{\varepsilon}{\rightarrow} \Delta \mathbb{Z} \rightarrow 0
$$

with the $P_{i}$ projective presheaves. Then the final term in (5) is the degree- $i$ cohomology of the cochain complex $\operatorname{Hom}_{\operatorname{PreSh}(\mathrm{C})}\left(P_{*}, F\right)$ :

$$
\cdots \stackrel{\delta^{*}}{\longleftarrow} \operatorname{Hom} \operatorname{PreSh}(\mathbf{C})\left(P_{1}, F\right) \stackrel{\delta^{*}}{\longleftarrow} \operatorname{Hom} \operatorname{PreSh}(\mathbf{C})\left(P_{0}, F\right) \longleftarrow 0
$$




\subsection{A projective resolution of $\Delta \mathbb{Z}$ and the Khovanov complex}

We return now to the particulars of Section 1.1 and compute the cochain complex (7) when $F=F_{K H}$, the Khovanov presheaf in $\operatorname{PreSh}(Q)$ where $Q$ is the poset of Section 1.1. To do this we present a particular projective resolution for the constant presheaf $\Delta \mathbb{Z}$ on $\boldsymbol{Q}$.

We start by constructing a presheaf $P_{n}$ in $\operatorname{PreSh}(Q)$ for each integer $n>0$. Remembering that $\boldsymbol{Q}$ is the cell poset of the regular CW complex $X$ of Section 1.1, for $x \in \boldsymbol{Q}$ set

$$
P_{n}(x):=\mathbb{Z}[n-\text { cells of } X \text { contained in the closure of the cell } x] .
$$

Thus if $\operatorname{dim} x<n$ then $P_{n}(x)=0$; if $\operatorname{dim} x=n$ then $P_{n}(x)=\mathbb{Z}[x] \cong \mathbb{Z}$; and if $\operatorname{dim} x>n$ then $P_{n}(x)$ is a direct sum of copies of $\mathbb{Z}$, one copy for each $n$-cell in the boundary of $x$. If $x \leq y$ in $Q$ then we take $P_{n}(x \leq y): P_{n}(y) \rightarrow P_{n}(x)$ to be the obvious inclusion.

For a given presheaf $F \in \operatorname{PreSh}(Q)$ there is a nice characterisation of the group of presheaf morphisms $P_{n} \rightarrow F$ :

Proposition 1.2 For $F \in \operatorname{PreSh}(Q)$ the map

$$
f^{n}: \operatorname{Hom}_{\operatorname{PreSh}(Q)}\left(P_{n}, F\right) \rightarrow \bigoplus_{\operatorname{dim} x=n} F(x)
$$

defined by $f^{n}(\tau)=\sum_{\operatorname{dim} x=n} \tau_{x}(x)$ is an isomorphism of abelian groups.

Proof That $f^{n}$ is a homomorphism is clear since $(\tau+\sigma)_{x}=\tau_{x}+\sigma_{x}$. To show injectivity, suppose that $f^{n}(\tau)=0$ from which it follows that $\tau_{x}(x)=0$ for all $n$-cells $x \in Q$. To show that $\tau=0$ we must prove that $\tau_{y}: P_{n}(y) \rightarrow F(y)$ is zero for all $y \in Q$. For $\operatorname{dim} y<n$ there is nothing to prove since $P_{n}(y)=0$. For $\operatorname{dim} y=n$ we have $P_{n}(y)=\mathbb{Z}[y]$ and $\tau_{y}(y)=0$ since $y$ is an $n$-cell. For $\operatorname{dim} y>n$,

$$
P_{n}(y)=\mathbb{Z}\left[y_{\alpha} \mid \operatorname{dim} y_{\alpha}=n \text { and } y_{\alpha} \text { in the closure of } y\right]
$$

and we have

$$
\tau_{y}\left(y_{\alpha}\right)=\tau_{y}\left(P_{n}\left(y \leq y_{\alpha}\right)\left(y_{\alpha}\right)\right)=F\left(y \leq y_{\alpha}\right)\left(\tau_{y_{\alpha}}\left(y_{\alpha}\right)\right)=0,
$$

the first equality since $P_{n}\left(y \leq y_{\alpha}\right): P_{n}\left(y_{\alpha}\right) \rightarrow P_{n}(y)$ is an inclusion, the second by naturality of $\tau$ and the third since $y_{\alpha}$ is an $n$-cell. Finally, $f^{n}$ is surjective because $P_{n}(x)$ is free and so there is no restriction on the images $\tau_{x}(x) \in F(x)$. 
The isomorphism given in Proposition 1.2 allows us to define a morphism $\tau: P_{n} \rightarrow F$ by specifying a tuple $\sum \lambda_{x} \in \bigoplus F(x)$, where the sum is over the $n$-cells $x$.

It is easy to see that the $P_{n}$ are projective presheaves. Given the following diagram of presheaves and morphisms (with solid arrows) and exact row:

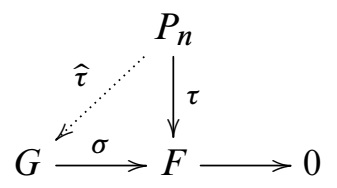

then the local maps $G(x) \stackrel{\sigma_{x}}{\longrightarrow} F(x)$ are surjections. Thus if $\sum \lambda_{x} \in \bigoplus F(x)$ specifies the map $\tau$ then for each $x$ there is a $\mu_{x} \in G(x)$ with $\sigma_{x}\left(\mu_{x}\right)=\lambda_{x}$. Hence there exists a morphism $\widehat{\tau}: P_{n} \rightarrow G$ specified by $\sum \mu_{x}$, which clearly makes the diagram commute. The $P_{n}$ are thus projective presheaves.

We now assemble the $P_{n}$ into a resolution of $\Delta \mathbb{Z}$ by defining maps $\delta_{n}: P_{n} \rightarrow P_{n-1}$. For $x \in Q$ let $\delta_{n, x}: P_{n}(x) \rightarrow P_{n-1}(x)$ be the homomorphism defined by

$$
\delta_{n, x}(y)=\sum_{y \prec z}[y, z] z
$$

for $y$ an $n$-cell $\subset \bar{x}$ and the sum being over the $(n-1)$-cells $z \subset \bar{y}$. Here, $[y, z]= \pm 1$ is the incidence number of $y$ and $z$ given by the orientations chosen at the end of Section 1.1. It is easy to check that these homomorphisms assemble into a morphism of presheaves $\delta_{n}: P_{n} \rightarrow P_{n-1}$. The sequence

$$
\cdots \stackrel{\delta}{\rightarrow} P_{n+1} \stackrel{\delta}{\rightarrow} P_{n} \stackrel{\delta}{\rightarrow} P_{n-1} \stackrel{\delta}{\rightarrow} \cdots
$$

is exact at $P_{n}$ if and only if each of the local sequences $P_{*}(x)$ is exact at $P_{n}(x)$. But $P_{*}(x)$ is nothing other than the cellular chain complex of the $\operatorname{dim}(x)$-dimensional ball corresponding to the closure of $x$ with the induced $\mathrm{CW}$ decomposition. In particular

$$
H_{n} P_{*}(x)= \begin{cases}\mathbb{Z} & n=0 \\ 0 & n>0\end{cases}
$$

so that $P_{*}(x)$, and hence $P_{*}$, is exact in degree $n>0$.

To define an augmentation $P_{0} \stackrel{\varepsilon}{\rightarrow} \Delta \mathbb{Z} \rightarrow 0$ take $\varepsilon$ to be the canonical surjection onto $\operatorname{coker}(\delta)$ :

$$
P_{1} \stackrel{\delta}{\rightarrow} P_{0} \stackrel{\varepsilon}{\rightarrow} \operatorname{coker}(\delta) \rightarrow 0
$$

The computation of $P_{*}(x)$ above immediately shows that $\operatorname{coker}(\delta) \cong \Delta \mathbb{Z}$.

We now have our projective resolution (6) for $\Delta \mathbb{Z}$ and hence a cochain complex (7) that computes the derived functors $\lim ^{i} F_{K H}$. Proposition 1.2 gives an isomorphism of 
graded abelian groups $f: \operatorname{Hom}_{\operatorname{PreSh}(Q)}\left(P_{*}, F_{K H}\right) \rightarrow K^{*}$ where $K^{*}$ is the Khovanov cochain complex of Section 1.1. As the following lemma shows, $f$ is in fact a chain map and thus there is an isomorphism of cochain complexes

$$
\operatorname{Hom}_{\operatorname{PreSh}(Q)}\left(P_{*}, F_{K H}\right) \cong K^{*} .
$$

Lemma 1.3 $f$ is a chain map $\operatorname{Hom}_{\operatorname{PreSh}(Q)}\left(P_{*}, F_{K H}\right) \rightarrow K^{*}$.

Proof We must show that the following diagram commutes.

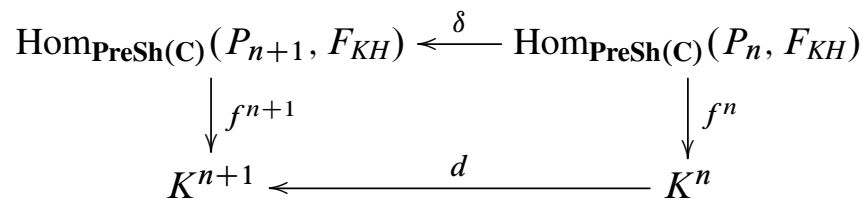

Let $\tau \in \operatorname{Hom}_{\operatorname{PreSh}(Q)}\left(P_{n}, F_{K H}\right)$ and write $F$ for $F_{K H}$. If $x$ is an $n$-cell, write $\lambda_{x}:=$ $\tau_{x}(x) \in F(x)$ so that $f^{n}$ sends $\tau$ to the tuple $\sum_{x} \lambda_{x}$, the sum over the $n$-cells of $X$. Applying the Khovanov differential $d$ we get

$$
d\left(f^{n}(\tau)\right)=\sum_{x} \sum_{y \prec x}[x, y] F(y \prec x)\left(\lambda_{x}\right) .
$$

Consider now $\delta(\tau)=\tau \delta \in \operatorname{Hom}_{\operatorname{PreSh}(\mathbf{C})}\left(P_{n+1}, F_{K H}\right)$. For $y$ an $(n+1)-$ cell we have $\delta_{y}(y)=\sum_{x \succ y}[x, y] x$ and by an argument similar to that in the proof of Proposition 1.2, for $x$ an $n$-cell we have $\tau_{y}(x)=F(y \prec x)\left(\lambda_{x}\right)$. Thus $f^{n+1}(\delta(\tau))$ is equal to

$$
\sum_{\operatorname{dim}}\left(\tau_{y} \delta_{y}\right)(y)=\sum_{y} \sum_{x \succ y}[x, y] \tau_{y}(x)=\sum_{y} \sum_{x \succ y}[x, y] F(y \prec x)\left(\lambda_{x}\right)=d\left(f^{n}(\tau)\right) .
$$

Summarising: to compute the higher limits of the Khovanov presheaf we use the complex $\operatorname{Hom}_{\operatorname{PreSh}(Q)}\left(P_{*}, F_{K H}\right)$, which is isomorphic to $K^{*}$, and this in turn computes the unnormalised Khovanov homology. We have therefore proved the first theorem:

Theorem 1.4 Let $D$ be a link diagram and let $F_{K H}: Q^{\text {op }} \rightarrow \mathbf{A b}$ be the Khovanov presheaf defined in Section 1.1. Then

$$
\overline{K H}^{i}(D) \cong{\overleftarrow{Q^{\mathrm{op}}}}_{\lim ^{i}} F_{K H}
$$

Remark It is essential that we use the modified Boolean lattice $\boldsymbol{Q}$ rather than just $\mathbf{B}$ : if we work with the Khovanov presheaf over $\mathbf{B}$ then the higher limits all vanish. This follows from the general fact that for a presheaf over a finite poset with unique maximal element the higher limits all vanish; see Mitchell [15]. 


\subsection{Aside on the cohomology of classifying spaces with coefficients in a presheaf}

Although not central to what follows it is worthwhile making the connection with a more topological description of higher limits in which $\lim ^{i} F_{K H}$ is identified with the cohomology of a classifying space equipped with a system of local coefficients. We recall that the classifying space $B \mathbf{C}$ is the geometric realisation of the nerve of the small category $\mathbf{C}$. This point of view is novel in the context of Khovanov homology, so we give a brief presentation of it, but otherwise we make no particular claim to originality here.

Starting with a presheaf $F \in \mathbf{P r e S h}(\mathbf{C})$, the cochain complex $C^{*}(B \mathbf{C}, F)$ is defined on the nerve of $\mathbf{C}$ to have cochains

$$
C^{n}(B \mathbf{C}, F)=\prod_{x_{0} \rightarrow \cdots \rightarrow x_{n}} F\left(x_{0}\right),
$$

the product over sequences of morphisms $x_{0} \stackrel{f_{1}}{\longrightarrow} \cdots \stackrel{f_{n}}{\longrightarrow} x_{n}$ in $\mathbf{C}$. If $\lambda \in C^{n}$ write $\lambda \cdot\left(x_{0} \rightarrow \cdots \rightarrow x_{n}\right)$ for the component of $\lambda$ in the copy of $F\left(x_{0}\right)$ indexed by the sequence $x_{0} \rightarrow \cdots \rightarrow x_{n}$. The coboundary map $d: C^{n}(B \mathbf{C}, F) \rightarrow C^{n+1}(B \mathbf{C}, F)$ is given by

$$
\begin{aligned}
& d \lambda \cdot\left(x_{0} \stackrel{f_{1}}{\longrightarrow} \cdots \stackrel{f_{n+1}}{\longrightarrow} x_{n+1}\right) \\
&=F\left(x_{0} \stackrel{f_{1}}{\longrightarrow} x_{1}\right)\left(\lambda \cdot\left(x_{1} \stackrel{f_{2}}{\longrightarrow} \cdots \stackrel{f_{n+1}}{\longrightarrow} x_{n+1}\right)\right) \\
& \quad+\sum_{i=1}^{n}(-1)^{i} \lambda \cdot\left(x_{0} \stackrel{f_{1}}{\longrightarrow} \cdots x_{i-1} \stackrel{f_{i} f_{i+1}}{\longrightarrow} x_{i+1} \cdots \stackrel{f_{n+1}}{\longrightarrow} x_{n+1}\right) \\
& \quad+(-1)^{n+1} \lambda \cdot\left(x_{0} \stackrel{f_{1}}{\longrightarrow} \cdots \stackrel{f_{n}}{\longrightarrow} x_{n}\right) .
\end{aligned}
$$

Write $H^{*}(B \mathbf{C}, F)$ for the cohomology of $C^{*}(B \mathbf{C}, F)$. The following result of Moerdijk [16, Proposition II.6.1] shows that this cochain complex computes the higher limits.

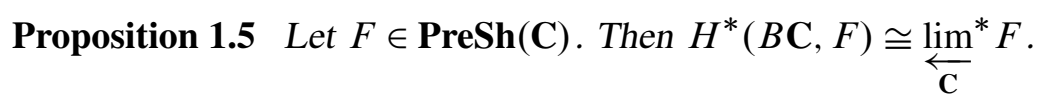

From Theorem 1.4 we immediately get the following description of unnormalised Khovanov homology in terms of the cohomology of the classifying space $B \boldsymbol{Q}$ with a system of local coefficients induced by the Khovanov presheaf:

Proposition 1.6 Let $D$ be a link diagram and let $F_{K H}: Q^{\text {op }} \rightarrow \mathbf{A b}$ be the Khovanov presheaf defined in Section 1.1. Then

$$
\overline{K H}^{*}(D) \cong H^{*}\left(B \boldsymbol{Q} ; F_{K H}\right) .
$$


Remark Proposition 1.6 is very similar in spirit to the main result of the authors' [6, Theorem 24], which gives an isomorphism between a homological version of Khovanov homology and a slight variation on the homology of a poset with coefficients in a presheaf (termed "coloured poset homology" in [6]; see also [7]).

\section{Interpreting higher limits in homotopy-theoretic terms}

\subsection{Homotopy limits}

Limits and colimits exist in the category of spaces but are problematic in the homotopy category: deforming the input data up to homotopy may not result in the same homotopy type. This problem is resolved by the use of homotopy limits and homotopy colimits, which are now standard constructions in homotopy theory. In this section we will use homotopy limits to build spaces whose homotopy groups are Khovanov homology. We begin by recalling the key properties of homotopy limits, and while we will adopt a black box approach to the actual construction (leaving the inner workings firmly inside the box), we will provide references to the classic text by Bousfield and Kan [3].

We briefly return to the generality of a small category, but later will again specialise to posets. Let $\mathbf{S p}$ denote the category of pointed spaces. All spaces from now on will be pointed. Let $\boldsymbol{C}$ be a small category and let $\mathbf{S p}^{C}$ be the category of diagrams of spaces of shape $\boldsymbol{C}:$ an object is a (covariant) functor $\mathscr{X}: \boldsymbol{C} \rightarrow \mathbf{S p}$ and a morphism $f: \mathscr{X} \rightarrow \mathscr{Y}$ is a natural transformation. Thus a diagram of spaces associates to each object of $\boldsymbol{C}$ a (pointed) space and to each morphism of $\boldsymbol{C}$ a (pointed) continuous function such that these fit together in a coherent way. Given a morphism $f: \mathscr{X} \rightarrow \mathscr{y}$ we will use the notation $f_{x}$ for the component at $x$. The trivial diagram takes as values the one-point space $\star$ for all objects of $\boldsymbol{C}$ and the identity map $\star \rightarrow \star$ for all morphisms.

For our purposes holim is a covariant functor

$$
\operatorname{holim}_{C}: \mathbf{S p}^{C} \rightarrow \mathbf{S p}
$$

whose main properties are recalled below in Propositions 2.1-2.5 . For a morphism $f: \mathscr{X} \rightarrow \mathscr{Y}$ we denote by $\bar{f}$ the induced map holim $\mathscr{X} \rightarrow$ holim $\mathscr{Y}$. The holim construction is natural with respect to change of underlying category: a functor $F: C^{\prime} \rightarrow C$ induces a map $\operatorname{holim}_{C} \mathscr{X} \rightarrow \operatorname{holim}_{\boldsymbol{C}}, \mathscr{X} \circ F$.

Remark We adopt the convention of Bousfield and Kan [3], where if pressed on the matter, space means "simplicial set". Furthermore, if thus pressed, we will also assume that diagrams take as values fibrant simplicial sets [3, VIII, 3.8]. Indeed there are models of Eilenberg-Mac Lane spaces that are simplicial groups, and hence fibrant. 
The reader should be aware however that in the proper generality the propositions below require fibrant objects.

The first important property of holim is its well-definedness in the homotopy category; it is robust with respect to deformation by homotopy [3, XI, 5.6]:

Proposition 2.1 (Homotopy) Let $f: \mathscr{X} \rightarrow \mathscr{Y}$ be a morphism in $\mathbf{S p}^{C}$ such that for all $x \in \mathscr{X}$ the map $f_{x}: \mathscr{X}(x) \rightarrow \mathscr{Y}(x)$ is a homotopy equivalence. Then

$$
\bar{f}: \operatorname{holim} \mathscr{X} \rightarrow \operatorname{holim} \mathscr{y}
$$

is a homotopy equivalence.

Next, a morphism of diagrams which is locally a fibration induces a fibration on holim $[3, \mathrm{XI}, 5.5]$ :

Proposition 2.2 (Fibration) Let $f: \mathscr{X} \rightarrow$ y be a morphism in $\mathbf{S p}^{\mathbf{C}}$ such that for all $x \in \mathscr{X}$ the map $f_{x}: \mathscr{X}(x) \rightarrow \mathscr{Y}(x)$ is a fibration. Then $\bar{f}:$ holim $\mathscr{X} \rightarrow \operatorname{holim} \mathscr{y}$ is a fibration.

There is also a nice description of holim for diagrams over a product of categories [ 3 , XI, 4.3]:

Proposition 2.3 (Product) Let $\mathscr{X}: \boldsymbol{C} \times \boldsymbol{D} \rightarrow \mathbf{S p}$ be a diagram of spaces over the product category $\boldsymbol{C} \times \boldsymbol{D}$. Then

$$
\operatorname{holim}_{C} \operatorname{holim}_{\boldsymbol{D}} \mathscr{X} \simeq \operatorname{holim}_{\boldsymbol{C} \times \boldsymbol{D}} \mathscr{X} \simeq \operatorname{holim}_{\boldsymbol{D}} \operatorname{holim}_{\boldsymbol{C}} \mathscr{X} .
$$

We also need to be able to compare diagrams of different shape, ie where the base categories are different. The result turns out to be easier to state in the context of posets than for small categories, and this suffices for us [3, XI, 9.2]:

Proposition 2.4 (Cofinality) Let $f: \boldsymbol{P}_{2} \rightarrow \boldsymbol{P}_{1}$ be a map of posets.

(i) Let $\mathscr{X}: \boldsymbol{P}_{1} \rightarrow \mathbf{S p}$ be a diagram of spaces and suppose that for any $x \in \boldsymbol{P}_{1}$ the poset $f^{-1}\left\{y \in \boldsymbol{P}_{1} \mid y \leq x\right\} \subset \boldsymbol{P}_{2}$ is contractible. Then holim $\boldsymbol{P}_{2} \mathscr{L} \circ f \simeq \operatorname{holim}_{\boldsymbol{P}_{1}} \mathscr{X}$.

(ii) Let $\mathscr{X}: \boldsymbol{P}_{1}^{\text {op }} \rightarrow \mathbf{S p}$ be a diagram of spaces and suppose that for any $x \in \boldsymbol{P}_{1}$ the poset $f^{-1}\left\{y \in \boldsymbol{P}_{1} \mid y \geq x\right\} \subset \boldsymbol{P}_{2}$ is contractible. Then holim $\boldsymbol{P}_{2}^{\text {op }} \mathscr{X} \circ f \simeq$ $\operatorname{holim}_{\boldsymbol{P}_{1}^{\text {op }}} \mathscr{X}$. 
Here a poset is contractible if its geometric realisation $B \boldsymbol{P}$ is contractible, so in particular $\boldsymbol{B} \boldsymbol{P}$, and hence $\boldsymbol{P}$, is nonempty. For example if $\boldsymbol{P}$ has an extremal (ie maximal or minimal) element then $B \boldsymbol{P}$ is a cone. Statement (ii) above is simply a restatement of (i), but the potential confusion in taking opposites makes it worth while stating both.

For a simple application of Proposition 2.4 let $\boldsymbol{P}_{2}$ be a contractible poset and $\mathscr{X}$ the constant diagram over $\boldsymbol{P}_{2}$ having as values the space $X$ at each $x$ and the identity map $X \rightarrow X$ at each morphism $x \rightarrow y$. Let $\boldsymbol{P}_{1}$ be the single element poset and $y$ the diagram having value $X$ at this single element. If $f: \boldsymbol{P}_{2} \rightarrow \boldsymbol{P}_{1}$ is the only possible map, then $\mathscr{X}=\mathscr{Y} \circ f$ and the conditions of Proposition 2.4 are satisfied. Thus holim $\mathscr{X} \simeq \operatorname{holim} \mathscr{Y} \simeq X$.

The final basic property of holim is that it commutes with mapping spaces (of pointed maps between pointed spaces); see [3, XI, 7.6]:

Proposition 2.5 (Mapping) Let $\mathscr{X}$ be a diagram of spaces in $\mathbf{S p}^{C}$ and let $Y$ be a (pointed) space. Then

$$
\operatorname{Map}(Y, \operatorname{holim} \mathscr{X}) \simeq \operatorname{holim} \operatorname{Map}(Y, \mathscr{X}) .
$$

Here $\operatorname{Map}(Y,-)$ is the functor that takes a pointed space $Z$ to the space of pointed maps from $Y$ to $Z$ and $\operatorname{Map}(Y, \mathscr{X}) \in \mathbf{S p}^{C}$ is the composition $\operatorname{Map}(Y,-) \circ \mathscr{X}$.

\subsection{Spaces for Khovanov homology}

Bousfield and Kan give an interpretation of derived functors of the inverse limit as follows. Consider the Eilenberg-Mac Lane functor $K(-, n): \mathbf{A b} \rightarrow \mathbf{S p}$ for which we adopt the construction given by Weibel $[19,8.4 .4]$ where there is an obvious choice of basepoint for $K(A, n)$. For more details on Eilenberg-Mac Lane spaces, see May [14, Chapter V] or Hatcher [9, Chapter 4]. The following proposition [3, XI, 7.2] gives an interpretation of $\lim ^{i} F$ in homotopy theoretic terms where $C$ is a small category.

Proposition 2.6 Let $F: C \rightarrow$ Ab be a (covariant) functor. Then there are natural isomorphisms

$$
\pi_{i}\left(\operatorname{holim}_{C} K(-, n) \circ F\right) \cong \begin{cases}\lim _{C}^{n-i} F & \text { if } 0 \leq i \leq n, \\ 0 & \text { else }\end{cases}
$$

The spaces $\operatorname{holim}_{C} K(-, n) \circ F$ contain no more information than higher derived functors of $F$. Indeed, as a consequence of the Dold-Kan theorem (see [19, Section 8.4] or Curtis [4, Section 5]) we have: 
Proposition 2.7 For $n$ big enough the space holim $C K(-, n) \circ F$ has the homotopy type of a product of Eilenberg-Mac Lane spaces:

$$
\operatorname{holim}_{C} K(-, n) \circ F \simeq \prod_{m} K\left(\lim _{C}^{n-m} F, m\right)
$$

For a self-contained and elementary argument proving the appropriate result needed here we refer the reader to [5].

After these preliminaries on homotopy limits we return to Khovanov homology. Associated to a link diagram $D$ we have the Khovanov presheaf $F_{K H}: Q^{\text {op }} \rightarrow \mathbf{A b}$ of Section 1.1. Let $n \in \mathbb{N}$ and let $\mathscr{F}_{n}: \boldsymbol{Q}^{\text {op }} \rightarrow \mathbf{S p}$ be the diagram of spaces defined by $\mathscr{F}_{n}=K(-, n) \circ F_{K H}$, the composition of $F_{K H}$ with the Eilenberg-Mac Lane space functor $K(-, n)$. We can now define a space $\boldsymbol{Y}_{n} D$ as the homotopy limit of this diagram:

$$
\boldsymbol{Y}_{n} D=\operatorname{holim}_{\boldsymbol{Q}^{\text {op }} \mathscr{F}_{n}}=\operatorname{holim}_{\boldsymbol{Q}^{\text {op }}} K(-, n) \circ F_{K H}
$$

Remark The homotopy limit above, taken over the augmented Boolean lattice $\boldsymbol{Q}$, is what Goodwillie [8], in his theory of calculus of functors, calls the total fibre of the (decorated) Boolean lattice B.

From Theorem 1.4 and Proposition 2.6 we see that $\boldsymbol{Y}_{n} D$ is a space whose homotopy groups are isomorphic to the unnormalised Khovanov homology of $D$ :

\section{Proposition 2.8}

$$
\pi_{i}\left(\boldsymbol{Y}_{n} D\right) \cong \begin{cases}\overline{K H}^{n-i}(D) & \text { if } 0 \leq i \leq n \\ 0 & \text { else }\end{cases}
$$

Indeed by Proposition 2.7 we have

$$
\boldsymbol{Y}_{n} D \simeq \prod_{m} K\left(\overline{K H}^{n-m}(D), m\right) .
$$

In order to normalise Khovanov homology a global degree shift is applied. As $\pi_{i} \Omega X \cong \pi_{i+1} X$ for a pointed space $X$, we see that degree shifts are implemented at the space level by taking loop spaces. Suppose now $D$ is oriented and has $c$ negative crossings. The collection of spaces $\boldsymbol{Y}_{*}=\left\{\boldsymbol{Y}_{n} D\right\}$ is an $\Omega$-spectrum which may be delooped $c$ times to obtain a new $\Omega$-spectrum $\boldsymbol{X}_{*} D=\Omega^{-c} \boldsymbol{Y}_{*} D$ whose homotopy groups are normalised Khovanov homology:

$$
\pi_{i}\left(X_{*} D\right) \cong K H^{-i}(D)
$$




\section{Diagrams over Boolean lattices and homotopy limits}

This section develops some results on the homotopy limits of diagrams defined over (modified) Boolean lattices. These will then provide tools applicable to Khovanov homology, and in the next section we illustrate this with homotopy theoretic proofs of some Khovanov homology results. In light of the remark after Proposition 2.7 some of the conclusions of this section are consequences of Goodwillie's calculus of functors, but we prefer to (re)prove the results we need in a self-contained manner.

We make extensive use of homotopy fibres and so record here some of their properties. Given a map (of pointed spaces) $f: X \rightarrow Y$ we define the homotopy fibre of $f$ as a homotopy limit by

$$
\operatorname{hofibre}(X \stackrel{f}{\longrightarrow} Y)=\operatorname{holim}(X \stackrel{f}{\longrightarrow} Y \longleftarrow \star) .
$$

By lifting the lid of the black box only a fraction (see [3, Chapter XI]) one sees that this has the homotopy type of the usual homotopy fibre: namely defining

(10) $E_{f}=\{(x, \alpha) \mid x \in X, \alpha:[0,1] \rightarrow Y$ a continuous map such that $\alpha(0)=f(x)\}$,

then this is a space homotopy equivalent to $X$ and the map $E_{f} \rightarrow Y$ sending $(x, \alpha) \mapsto \alpha(1)$ is a fibration whose fibre is homotopy equivalent to the hofibre (9).

Relevant examples of homotopy fibres are

$$
\begin{aligned}
& \operatorname{hofibre}(X \rightarrow \star) \simeq X, \\
& \operatorname{hofibre}(\star \rightarrow Y) \simeq \Omega Y .
\end{aligned}
$$

Using the long exact homotopy sequence for a fibration and the Whitehead theorem one immediately gets:

Lemma 3.1 For $Y$ connected, if hofibre $(X \rightarrow Y) \simeq \star$ then $X \simeq Y$.

If $f: X \rightarrow Y$ is a map of pointed spaces with $Y$ contractible, then (11) extends to

$$
\operatorname{hofibre}(X \stackrel{f}{\longrightarrow} Y) \simeq X,
$$

and similarly if $X$ is contractible then (12) extends to

$$
\operatorname{hofibre}(X \stackrel{f}{\longrightarrow} Y) \simeq \Omega Y \text {. }
$$

From now on we assume that $\mathbf{C}$ is a connected category. Given diagrams $\mathscr{X}, \mathscr{Y} \in \mathbf{S p}^{C}$ (of pointed spaces) and a morphism $f: \mathscr{X} \rightarrow \mathscr{Y}$ one may form the homotopy fibre diagram $\operatorname{hof}(f)$ by (locally) defining $\operatorname{hof}(f)(x)=\operatorname{hofibre}\left(f_{x}: \mathscr{L}(x) \rightarrow \mathscr{Y}(x)\right)$ and 
$\operatorname{hof}(f)(x \rightarrow y)$ : hofibre $\left(f_{x}\right) \rightarrow$ hofibre $\left(f_{y}\right)$ the map induced by taking homotopy limits of the two rows of the diagram

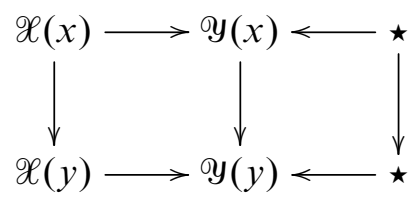

with the left-hand square commuting courtesy of $f$. It is a standard trick in homotopy theory to compute the homotopy limit of a diagram of homotopy fibres as a homotopy fibre. In the interest of completeness we have included the details, but the main point is that the homotopy fibre is an example of a homotopy limit and homotopy limits enjoy the product property of Proposition 2.3:

Proposition 3.2 Let $f: \mathscr{X} \rightarrow$ y be a morphism in $\mathbf{S p}^{C}$. Then

$$
\operatorname{holim}(\operatorname{hof}(f)) \simeq \operatorname{hofibre}(\operatorname{holim} \mathscr{X} \stackrel{\bar{f}}{\longrightarrow} \operatorname{holim} \mathscr{Y}) \text {. }
$$

Proof Let $\boldsymbol{D}$ be the three element category $a \stackrel{\alpha}{\longrightarrow} c \stackrel{\beta}{\longleftarrow} b$. Define $\mathscr{L}: \boldsymbol{C} \times \boldsymbol{D} \rightarrow \mathbf{S p}$ by

$$
\begin{aligned}
& \mathscr{L}(x, a)=\star, \\
& \mathscr{L}(x, b)=\mathscr{L}(x), \\
& \mathscr{L}(x, c)=\mathscr{y}(x) .
\end{aligned}
$$

On morphisms let

$$
\begin{aligned}
& \mathscr{L}((\mathrm{id}, \alpha):(x, a) \rightarrow(x, c))=\star \rightarrow \mathscr{Y}(x), \\
& \mathscr{L}((\mathrm{id}, \beta):(x, b) \rightarrow(x, c))=f_{x},
\end{aligned}
$$

and

$$
\mathscr{L}\left((\theta, z \stackrel{1}{\rightarrow} z):(x, z) \rightarrow\left(x^{\prime}, z\right)\right)= \begin{cases}\star \rightarrow \star, & z=a, \\ \mathscr{X}(\theta), & z=b, \\ \mathscr{y}(\theta), & z=c .\end{cases}
$$

We then have

$$
\begin{aligned}
\operatorname{holim}_{\boldsymbol{D}} \mathscr{L}(x,-) & =\operatorname{holim}\left(\mathscr{L}(x) \stackrel{f_{x}}{\longrightarrow} \mathscr{y}(x) \longleftarrow \star\right) \\
& =\operatorname{hofibre}\left(f_{x}: \mathscr{X}(x) \rightarrow \mathscr{y}(x)\right) \\
& =\operatorname{hof}(f)(x)
\end{aligned}
$$

from which we get $\operatorname{holim}_{C} \operatorname{holim}_{\boldsymbol{D}} \mathscr{\mathscr { E }} \simeq \operatorname{holim} \operatorname{hof}(f)$. Going the other way we have $\operatorname{holim}_{C} \mathscr{L}(-, a) \simeq \star, \operatorname{holim}_{C} \mathscr{L}(-, b)=\operatorname{holim}_{C} \mathscr{X}$, and $\operatorname{holim}_{C} \mathscr{L}(-, c)=\operatorname{holim}_{\boldsymbol{C}} \mathscr{Y}$, 
So

$$
\begin{aligned}
\operatorname{holim}_{\boldsymbol{D}} \operatorname{holim}_{\boldsymbol{C}} \mathscr{L} & =\operatorname{holim}(\operatorname{holim} \mathscr{X} \stackrel{\bar{f}}{\longrightarrow} \operatorname{holim} \mathscr{y} \longleftarrow \star) \\
& \simeq \operatorname{hofibre}(\operatorname{holim} \mathscr{X} \stackrel{\bar{f}}{\longrightarrow} \operatorname{holim} \mathscr{y}) .
\end{aligned}
$$

The result now follows from Proposition 2.3.

Later we will use this result in the form: if $f: \mathscr{L} \rightarrow \mathscr{Y}$ is a map of diagrams, the space holim $y$ is connected and holim hof $(f)$ contractible, then the induced map $\bar{f}: \operatorname{holim} \mathscr{X} \rightarrow \operatorname{holim} \mathscr{Y}$ is a homotopy equivalence.

Notation for diagrams of spaces We introduce a convenient notation that we will use extensively. We recall that space means pointed space and diagrams of spaces take values in pointed spaces. A Boolean lattice $\mathbf{B}$ will be represented by the circle below left and a diagram $\mathscr{X}: \mathbf{B}^{\text {op }} \rightarrow \mathbf{S p}$ by the pictogram below right:

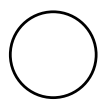

Extending this to $\boldsymbol{Q}^{\text {op }}$ by defining $\mathscr{L}\left(\mathbf{1}^{\prime}\right)=\star$ we obtain a diagram of spaces, with $\boldsymbol{Q}$ and $\mathscr{X}: \boldsymbol{Q}^{\mathrm{op}} \rightarrow \mathrm{Sp}$ represented as:
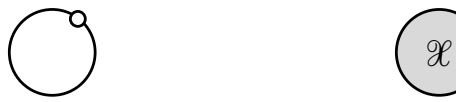

The trivial diagram will be denoted by

Let $\mathbf{B}=\mathbf{B}_{A}$ be Boolean of rank $r$, ie the lattice of subsets of $\{1, \ldots, r\}$. For each $1 \leq k \leq r$ there is a splitting of $\mathbf{B}$ into two subposets, both isomorphic to Boolean lattices of rank $r-1$ : one consists of those subsets containing $k$ and the other of those not containing $k$. Below we see the splittings for $r=3$, with (from left to right) $k=1,2$ and 3 :
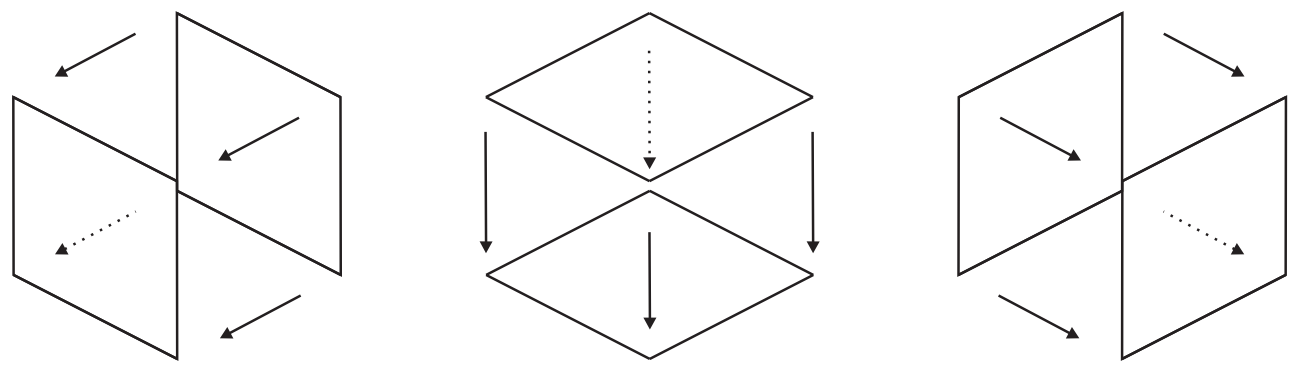
A diagram $\mathscr{X}: \mathbf{B}^{\mathrm{op}} \rightarrow \mathbf{S p}$ determines (and is determined by) two diagrams of spaces $\mathscr{X}_{1}$ and $\mathscr{L}_{2}$ over these rank $r-1$ Boolean lattices along with a morphism of diagrams $f: \mathscr{X}_{1} \rightarrow \mathscr{X}_{2}$. We denote this situation (and the obvious extension to $\boldsymbol{Q}^{\mathrm{op}}$ ) by the pictograms:
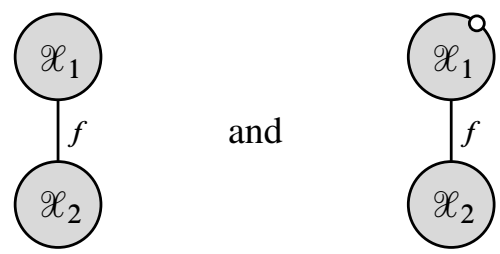

This process can be iterated with each of the smaller Boolean lattices to give pictures that are square, cubical, etc.

Lemma 3.3 Let $\mathscr{X}: \mathbf{B}^{\text {op }} \rightarrow \mathbf{S p}$ be a diagram of spaces. Then:

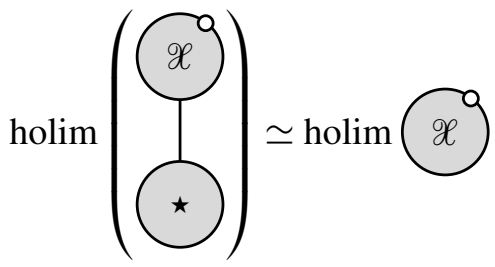

Proof If $\mathbf{B}$ has rank $r$ then the diagram on the left-hand side is over a Boolean lattice of rank $r+1$. Let $\boldsymbol{Q}$ be the extended version of this Boolean lattice and suppose that it has been split as above. Collapsing the bottom Boolean lattice to a point we obtain a new poset $\boldsymbol{P}$ :

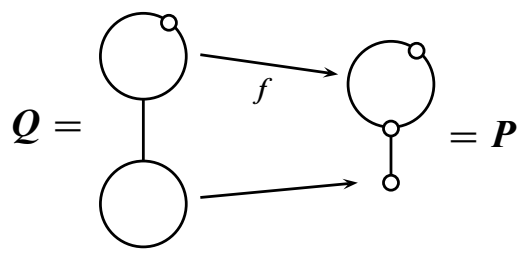

The poset map $f: \boldsymbol{Q} \rightarrow \boldsymbol{P}$ which collapses the lower Boolean lattice to a single point satisfies the hypotheses of Proposition 2.4 (ii). Moreover we have the following equality of diagrams of spaces (of shape $\boldsymbol{Q}^{\mathrm{op}}$ ):

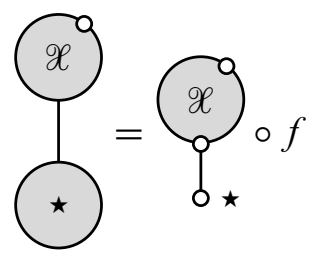


Let $\boldsymbol{Q}^{\prime}$ be the poset obtained from $\boldsymbol{Q}$ by removing the lower Boolean lattice; then the obvious inclusion $i: \boldsymbol{Q}^{\prime} \rightarrow \boldsymbol{P}$ satisfies the hypotheses of Proposition 2.4. Hence we have

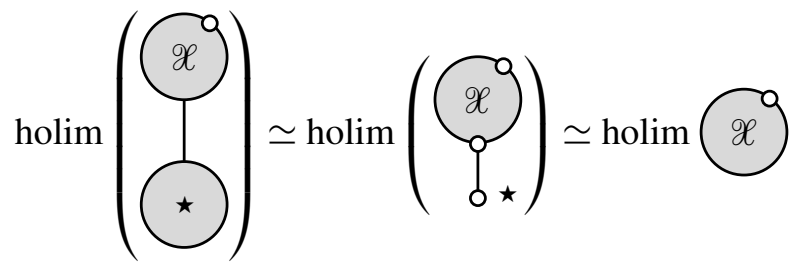

using Proposition 2.4 twice (with $f$ and $i$ ).

A somewhat more general version of this result is the following.

Lemma 3.4 Let $\mathscr{X}, \mathscr{Y}: \mathbf{B}^{\mathrm{op}} \rightarrow \mathbf{S p}$ be diagrams of spaces such that $\mathscr{Y}(x)$ is contractible for all $x \in \mathbf{B}^{\text {op }}$. Then:

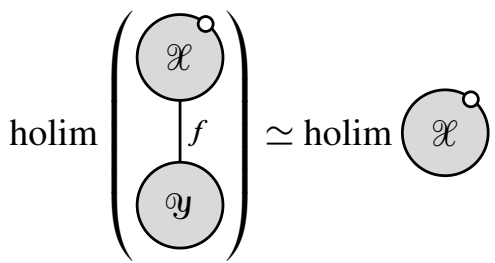

Proof Let $\tau$ be the morphism of diagrams defined by:

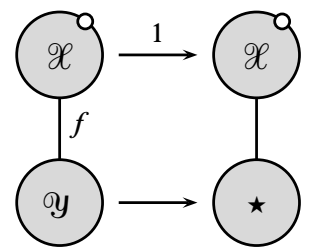

As the map $\mathscr{Y}(x) \rightarrow \star$ is a homotopy equivalence for all $x$, the result follows from Proposition 2.1 and Lemma 3.3.

Lemma 3.5 Let $\mathscr{X}: \mathbf{B}^{\text {op }} \rightarrow$ Sp be a diagram of spaces. Then:

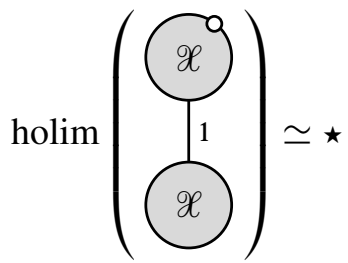

Proof Let $\boldsymbol{Q}$ be an extended Boolean lattice of rank one bigger than the rank of $\mathbf{B}$ and split as above. Let $\boldsymbol{P}$ be obtained from $\mathbf{B}$ by adding an element $\mathbf{1}^{\prime \prime}$ which is greater than all other elements (including the existing maximal element in $\mathbf{B}$ ). Pictorially: 


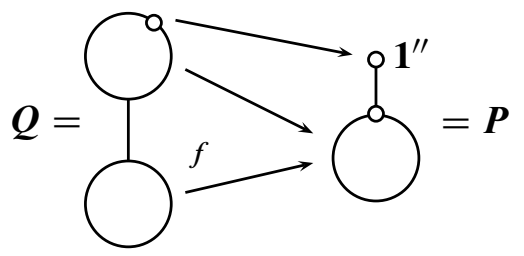

The poset map $f: \boldsymbol{Q} \rightarrow \boldsymbol{P}$ which identifies elements of the Boolean lattices and sends $\mathbf{1}^{\prime} \mapsto \mathbf{1}^{\prime \prime}$ satisfies the hypotheses of Proposition 2.4. Moreover we have the following equality of diagrams of spaces (of shape $\boldsymbol{Q}^{\mathrm{op}}$ ).

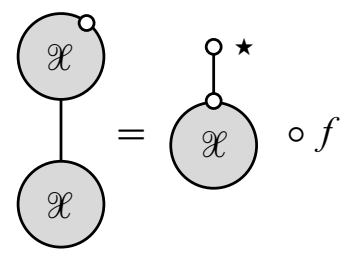

Since $\boldsymbol{P}^{\text {op }}$ has a minimal element it follows from [3, XI, 4.1 (iii)] that

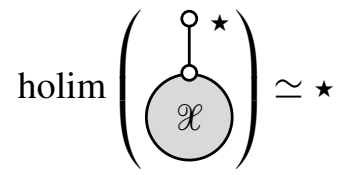

whence the result on applying Proposition 2.4.

Proposition 3.6 Let $f:(x) \rightarrow$ be a morphism of diagrams of spaces over a Boolean lattice. Then

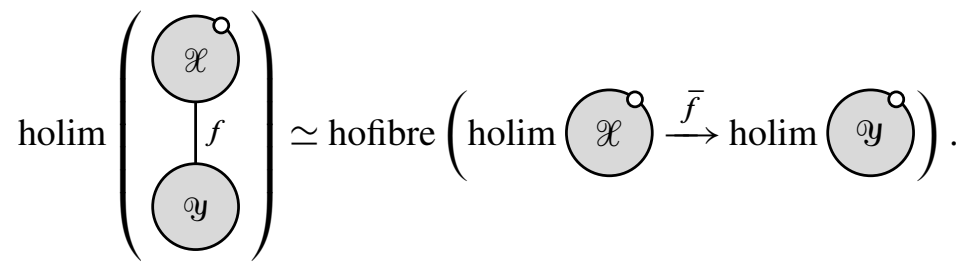

Proof Let $g$ be the following morphism of diagrams of spaces:

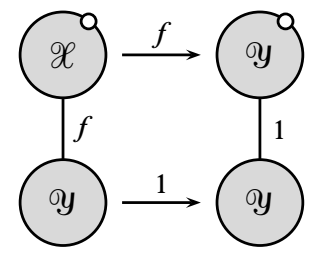


We have $\operatorname{holim}(\operatorname{hof}(g))$ is homotopy equivalent to

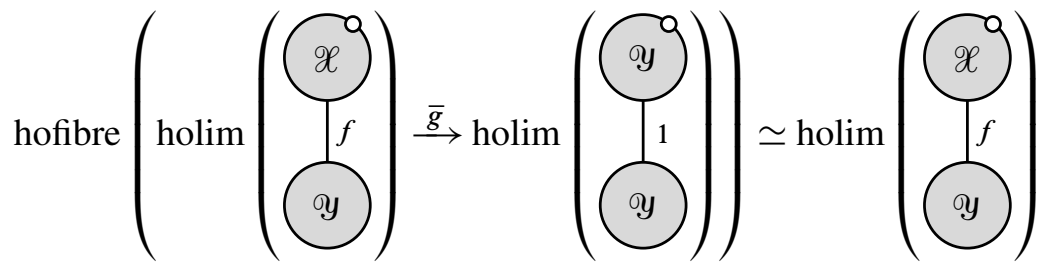

by Proposition 3.2, Lemma 3.5 and (13). On the other hand, writing

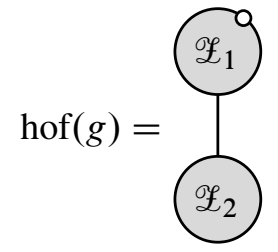

we see that $\mathscr{L}_{2}(x)$ is contractible for all $x$. Thus, by Lemma 3.4 and Proposition 3.2 we have

$$
\operatorname{holim}(\operatorname{hof}(g)) \simeq \operatorname{holim}\left(\mathscr{L}_{1} 9 \simeq \operatorname{hofibre}(\operatorname{holim} \mathscr{x} \stackrel{\bar{f}}{\rightarrow} \operatorname{holim}(99) .\right.
$$
Corollary 3.7 Let $f: \mathscr{Q} \rightarrow(9$ be a morphism of diagrams of spaces over a
Boolean lattice. Then

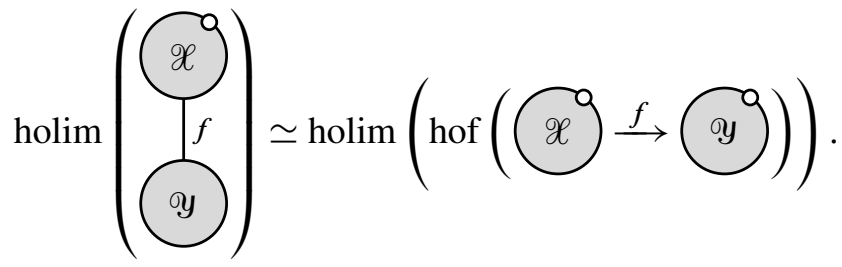

Lemma 3.3 can be generalised as in the first part of the following:

Proposition 3.8 Let $\mathscr{Y}: \mathbf{B}^{\mathrm{op}} \rightarrow \mathbf{S p}$ be a diagram of spaces with holim Then:

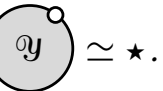

(i) holim

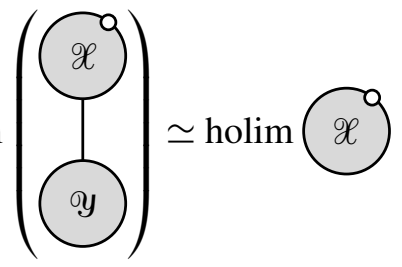

(ii) holim

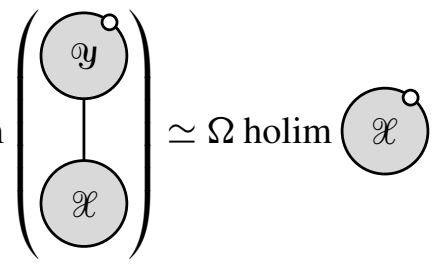


Proof For (ii) we have

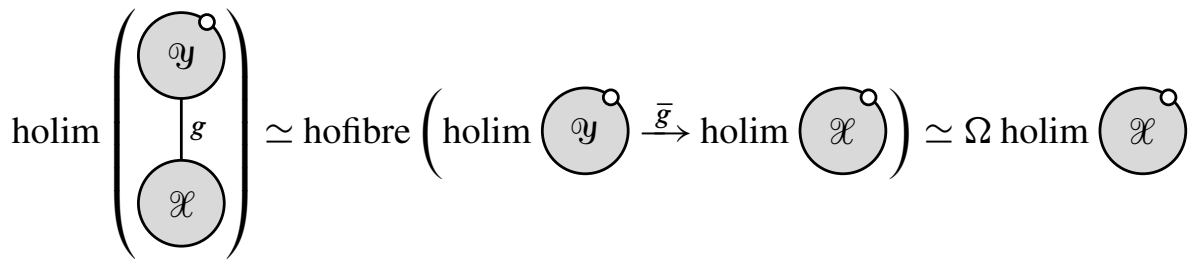

by Proposition 3.6 and Equation (14). Part (i) is similar.

Corollary 3.9 Let $\mathscr{X}, \mathscr{Y}: \mathbf{B}^{\mathrm{op}} \rightarrow \mathbf{S p}$ be diagrams of spaces such that $\mathscr{Y}(x)$ is contractible for all $x \in \mathbf{B}^{\text {op }}$. Then,

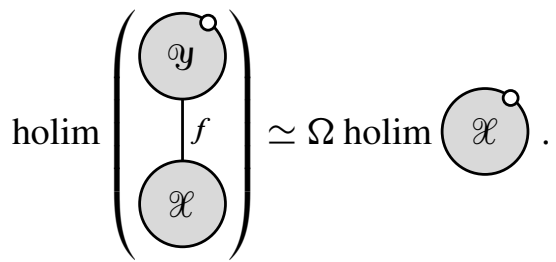

Proof Let $\tau$ be the morphism of diagrams defined by:

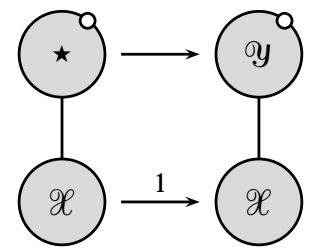

As the map $\star \rightarrow \mathscr{Y}(x)$ is a homotopy equivalence for all $x$, the result follows from Propositions 2.1 and 3.8 (ii).

Notation for presheaves We adopt a similar notation for presheaves to that for diagrams of spaces, with the difference that the circles are white rather than shaded. Thus a presheaf $F: \mathbf{B}^{\text {op }} \rightarrow \mathbf{A b}$ and its extension $F: Q^{\text {op }} \rightarrow \mathbf{A b}\left(\right.$ with $F\left(\mathbf{1}^{\prime}\right)=0$ ) will be represented by:

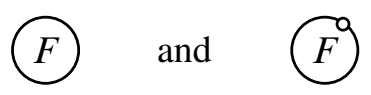

Given a presheaf $F$ we will denote the diagram of spaces $K(-, n) \circ F$ by $F$.

Remark For $n$ sufficiently large the space holim $F$ is path connected. To see this, one may use Proposition 2.6 to calculate

$$
\pi_{0}(\operatorname{holim} F) \cong \lim ^{n} F .
$$


So long as $n$ is chosen to be greater than the rank of $\boldsymbol{Q}$ the right-hand side is trivial by Theorem 1.4 (recall that the underlying poset is always assumed connected). We will always assume that $n$ is large enough in this sense.

Proposition 3.10 Let $F \stackrel{f}{\rightarrow} G \stackrel{g}{\rightarrow}(H$ be a short exact sequence in $\operatorname{PreSh}(Q)$. Then:

(i) hofibre $(\operatorname{holim} G \stackrel{\bar{g}}{\rightarrow} \operatorname{holim}(H) \simeq \operatorname{holim}(F$

(ii) hofibre $(\operatorname{holim}(F) \stackrel{\bar{f}}{\rightarrow} \operatorname{holim}(G) \simeq \Omega$ holim $H$

Proof For part (i) the left-hand side is homotopy equivalent to $\operatorname{holim}(\operatorname{hof}(g))$ by Proposition 3.2. Here $\operatorname{hof}(g)$ is the diagram obtained by taking homotopy fibres after applying $K(-, n)$ to the surjection $g$ in the statement of the proposition, so $\operatorname{hof}(g)(x)=\operatorname{hofibre}(K(G(x), n) \rightarrow K(H(x), n))$. We can identify this homotopy fibre with $K\left(\operatorname{ker}\left(g_{x}\right), n\right)=K(F(x), n)$. The maps in the diagram $\operatorname{hof}(g)$ under this identification correspond to the maps induced by $F$; this follows since maps on such Eilenberg-Mac Lane spaces are completely determined by their effect on $\pi_{n}$ and by the naturality of the hofibre construction. Thus the diagram $\operatorname{hof}(g)$ is equivalent to F and the result follows by Proposition 2.1. Part (ii) is similar.

Combining Proposition 3.10, Lemma 3.1 and the remark preceding Proposition 3.10 gives the following very useful lemma.

Corollary 3.11 Let $F \stackrel{f}{\rightarrow} G \stackrel{g}{\rightarrow}(H)$ be a short exact sequence in $\operatorname{PreSh}(Q)$. Then:

(i) If holim $F \simeq \star$ then $\bar{g}: \operatorname{holim} G \simeq$ holim $H$ is a homotopy equivalence.

(ii) If holim $H \simeq \star$ then $\bar{f}$ : holim $F \simeq$ holim $G$ is a homotopy equivalence.

Another result that will prove useful comes from combining Propositions 3.10 and 3.6:

Corollary 3.12 Let $F \stackrel{f}{\rightarrow} G \stackrel{g}{\rightarrow}(H)$ be a short exact sequence in $\operatorname{PreSh}(Q)$. Then:

(i) $\operatorname{holim}(\underbrace{G}_{g}) \simeq \operatorname{holim}(F)$ (ii) $\operatorname{holim}(\underbrace{}_{f}) \simeq \Omega \operatorname{holim}(H$ 


\section{Applications to Khovanov homology}

The results of the previous section give a collection of tools for Khovanov homology, and in this section we illustrate it with a few simple examples. First we isolate the two most useful results. One is Corollary 3.11, which is just a presheaf theoretic reformulation of standard arguments involving chain complexes:

\section{Presheaf computational tool Let}

$$
\text { (H) } \rightarrow \text { F } \rightarrow G
$$

be a short exact sequence. If holim $(H)$ resp. holim $G)$ is contractible then

$$
\operatorname{holim} F \simeq \operatorname{holim} G(\operatorname{resp} \operatorname{holim}(H \simeq \operatorname{holim} F) \text {. }
$$

An arbitrary morphism of presheaves (not necessarily injective or surjective) cannot be slotted into a short exact sequence. Our second result, which follows from Proposition 3.2 and the discussion preceeding it, along with the remark right before Proposition 3.10, gets around this by using the homotopy theory in a more essential way:

Homotopy computational tool Let $F \rightarrow G$ be a morphism in $\operatorname{PreSh}(Q)$ with

$$
\mathscr{H}=\operatorname{hof}(F) \rightarrow G) \text {. }
$$

If holim $\mathscr{H}$ (resp. holim G $)$ is contractible then

$$
\operatorname{holim}(F \simeq \operatorname{holim} G(\operatorname{resp} \operatorname{holim} \mathscr{H} \simeq \operatorname{holim}(F) .
$$

Notation for the Khovanov presheaf of a link diagram We extend our notation to the specific case of the Khovanov presheaf $F_{K H}$ associated to a link diagram. It is convenient to have the link diagram back in the picture: given a link diagram $D$ we denote the associated Khovanov presheaf $F_{K H}$ and diagram of spaces $K(-, n) \circ F_{K H}$ by:

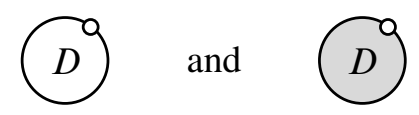

If, as is often the case, we are interested in a link diagram with a specified local piece we simply display it inside the circle. Thus for example the unit map $\iota: \mathbb{Z} \rightarrow V$, which 
is defined by $\iota(1)=1$, extends to an injective morphism of presheaves over a Boolean lattice of appropriate rank which we denote by:

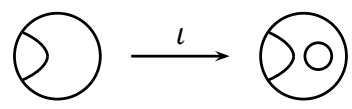

We have link diagrams $D$ and $D^{\prime}$ that are identical outside of one part where they differ by the local piece shown. On the left we have the presheaf $F_{K H}: \mathbf{B}^{\mathrm{op}} \rightarrow \mathbf{A b}$, the cube for $D$, and on the right $F_{K H}^{\prime}: \mathbf{B}^{\text {op }} \rightarrow \mathbf{A b}$, the cube for $D^{\prime}$. For $x \in \mathbf{B}$ we have $F_{K H}^{\prime}(x)=F_{K H}(x) \otimes V$ and the map

$$
\iota_{x}: F_{K H}(x)=F_{K H}(x) \otimes \mathbb{Z} \rightarrow F_{K H}(x) \otimes V=F_{K H}^{\prime}(x)
$$

is the map $1 \otimes \iota$. These local $\iota_{x}$ stitch together to form a morphism of presheaves $\iota: F_{K H} \rightarrow F_{K H}^{\prime}$. This is what we mean by the picture above.

Similarly the counit map $\epsilon: V \rightarrow \mathbb{Z}$, defined by $\epsilon(1)=0, \epsilon(u)=1$, extends to a surjective morphism of presheaves over $\mathbf{B}$

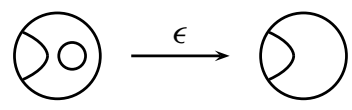

and there is a short exact sequence of presheaves

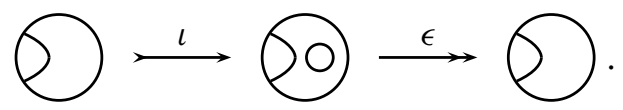

The multiplication $m: V \otimes V \rightarrow V$ is surjective with $\operatorname{ker}(m) \cong V$, and this analysis similarly extends to give a short exact sequence of presheaves

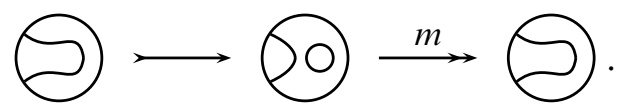

The composition $m \circ \iota$ is the identity map. Finally, the comultiplication $\Delta: V \rightarrow V \otimes V$ is injective, $\operatorname{coker}(\Delta) \cong V$, and this extends to a short exact sequence of presheaves

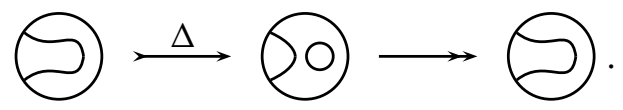

The composition $\epsilon \circ \Delta$ is the identity map.

Occasionally the link diagram $D$ will be too large for the circle notation above (eg in Section 4.3), and so we will just write $D$, or a shaded version. For example if $D_{1}, D_{2}$ are unoriented link diagrams then (15) extends to

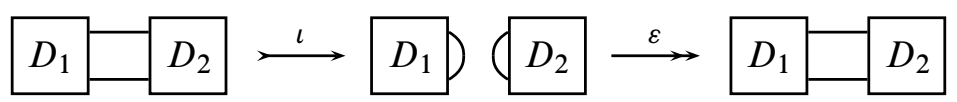

and there are similar sequences for $m$ and $\Delta$. 


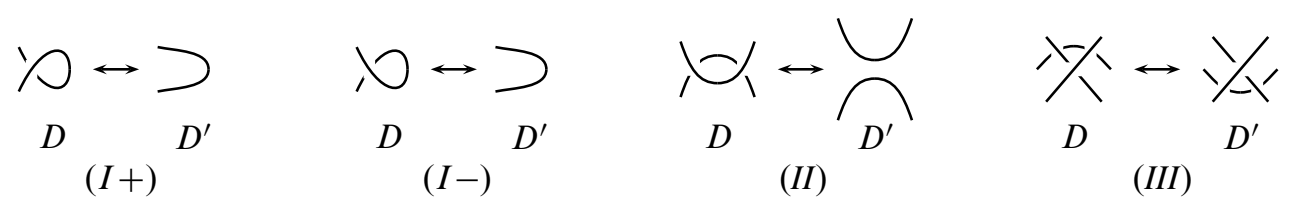

Figure 2: Reidemeister moves

\subsection{The skein relation}

By choosing a crossing there is an evident smoothing change morphism of presheaves:

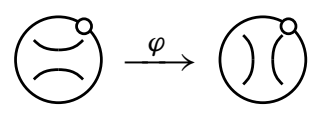

In general this is neither surjective nor injective. There is however an induced map of spaces

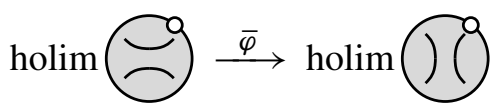

and we can easily describe its homotopy fibre to give a homotopy theoretic incarnation of the skein relation:

Proposition 4.1 hofibre $(\operatorname{holim}(9) \rightarrow \operatorname{holim}(9) \simeq \operatorname{holim} \times 9$.

Proof We have

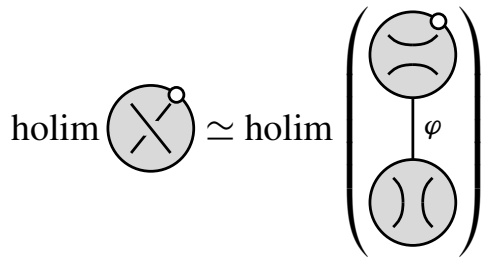

and the result follows immediately from Proposition 3.6.

The associated long exact homotopy sequence can be identified with the usual long exact skein sequence in Khovanov homology (see [18] and [17]).

\subsection{Reidemeister invariance.}

We now give a homotopy theoretic proof of the invariance of Khovanov homology by Reidemeister moves (Figure 2). The original proofs can be found in [10] (see also [1]) and more a geometrical argument can be found in [2]. We recall that the rank of the underlying Boolean lattice is the number of crossings in the given diagram, thus moves $(I \pm)$ and $(I I)$ alter the underlying Boolean lattice. 
Recalling from the remarks at the end of Section 2.2 that a negative degree shift in Khovanov homology is equivalent to taking the loop space, we see that we must prove $(I+) \boldsymbol{Y}_{n} D \simeq \boldsymbol{Y}_{n} D^{\prime},(I-) \boldsymbol{Y}_{n} D \simeq \Omega \boldsymbol{Y}_{n} D^{\prime}$, (II) $\boldsymbol{Y}_{n} D \simeq \Omega \boldsymbol{Y}_{n} D^{\prime}$, (III) $\boldsymbol{Y}_{n} D \simeq \boldsymbol{Y}_{n} D^{\prime}$.

4.2.1 Reidemeister moves $(I \pm)$. Let $D$ and $D^{\prime}$ be two (unoriented) link diagrams locally described as in $(I-)$ in Figure 2. The short exact sequence (17) and Corollary 3.12 (ii) give

$$
Y_{n} D=\operatorname{holim}(\underbrace{}_{\Delta}=\Omega \operatorname{holim} \bigcirc=\Omega Y_{n} D^{\prime} .
$$

A very similar argument, using (16) and Corollary 3.12 (i), gives Reidemeister $(I+)$. 4.2.2 Reidemeister move (II). Let $D$ and $D^{\prime}$ be two link diagrams locally described as in $(I I)$ and let $F_{K H}$ be the Khovanov presheaf for $D$. There is a short exact sequence $H \succ F_{K H} \rightarrow G$ :

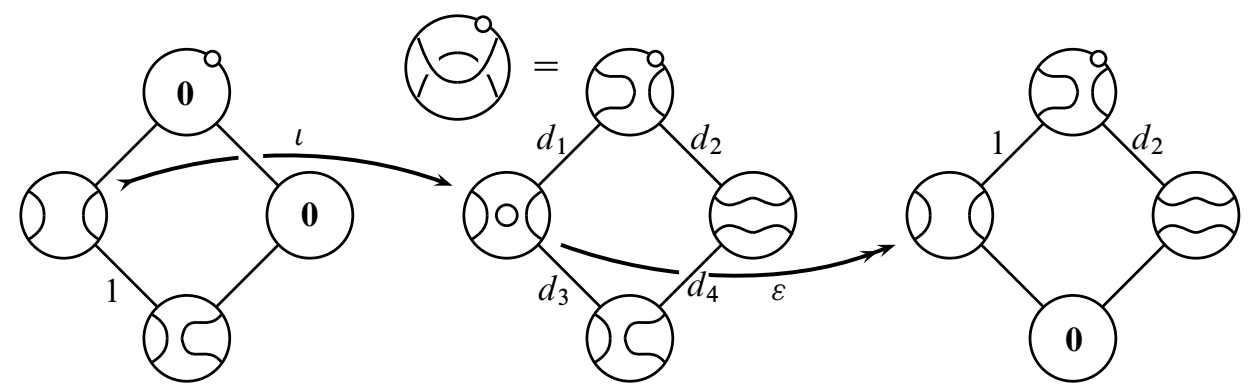

We leave it to the reader to check that $G$ and $H$ are indeed presheaves. All missing horizontal maps are either the identity or zero (it should be clear which is which), $\iota$ and $\epsilon$ are the unit and counit, and we are using the short exact sequence (15). To check that we have morphisms of presheaves we need to show that $\varepsilon d_{1}=1$ and $d_{3} \iota=1$. The former follows from $\varepsilon \Delta=1$ and the latter from $m \iota=1$.

We have

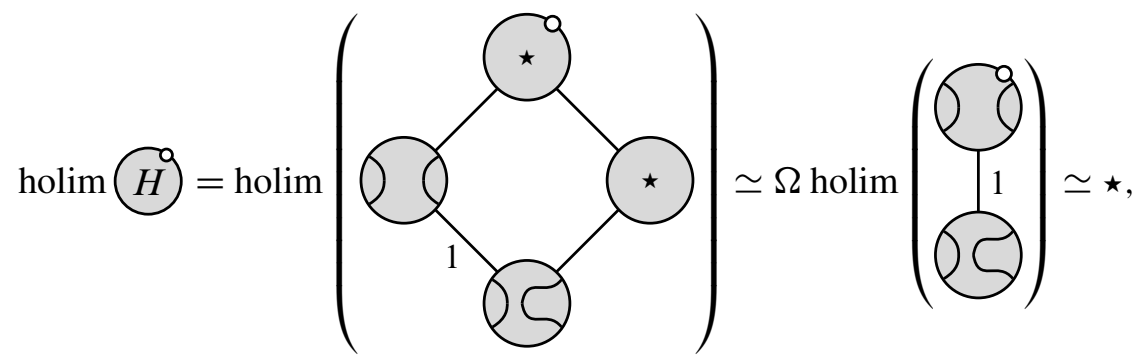


with the homotopy equivalences by Propositions 3.10 (i), 3.8 and Lemma 3.5 respectively. The map induced by $F_{K H} \rightarrow G$ is thus a homotopy equivalence by the presheaf computational tool, and so $\boldsymbol{Y}_{n} D$ equals

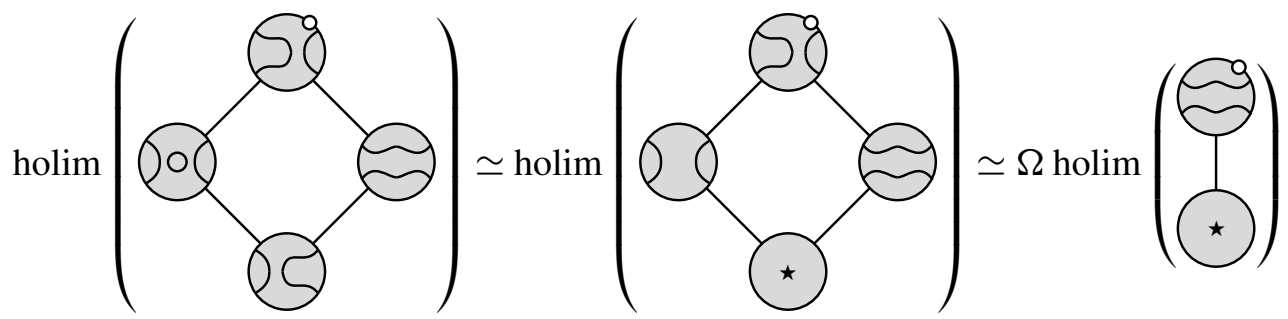

with the last homotopy equivalent to $\Omega$ holim $=\Omega Y_{n} D^{\prime}$ by Proposition 3.8 (ii) and Lemma 3.3.

4.2.3 Reidemeister move (III). Let $D$ and $D^{\prime}$ be two (unoriented) link diagrams locally described as in (III) in Figure 2 and let $F_{K H}$ be the Khovanov presheaf for $D$. We start with a short exact sequence $G_{0} \succ F_{K H} \stackrel{\tau}{\longrightarrow} G$ defined by:

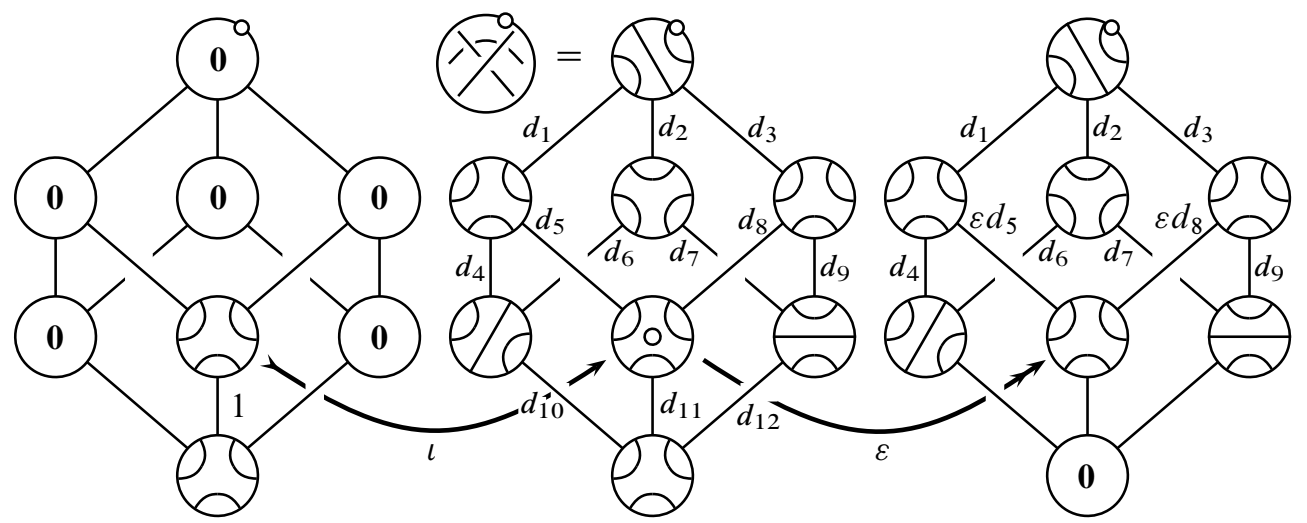

One can check that $G$ is indeed a presheaf and that $\tau$ is surjective. All missing horizontal maps are either the identity or zero. Proposition 3.8 (ii) gives

$$
\operatorname{holim} G_{0}^{9} \simeq \Omega^{2} \star \star \star,
$$

hence holim $F \simeq$ holim $G$ by the presheaf tool (abbreviating $F_{K H}$ to $F$ ).

We now define another presheaf $H$ and a presheaf map $\sigma: G \rightarrow H$ where all missing maps are either the identity or zero as before. For this we note that in $G$ we have $\varepsilon d_{5}=\varepsilon d_{8}=\Delta \varepsilon=1$ and $d_{1}=d_{3}$ : 


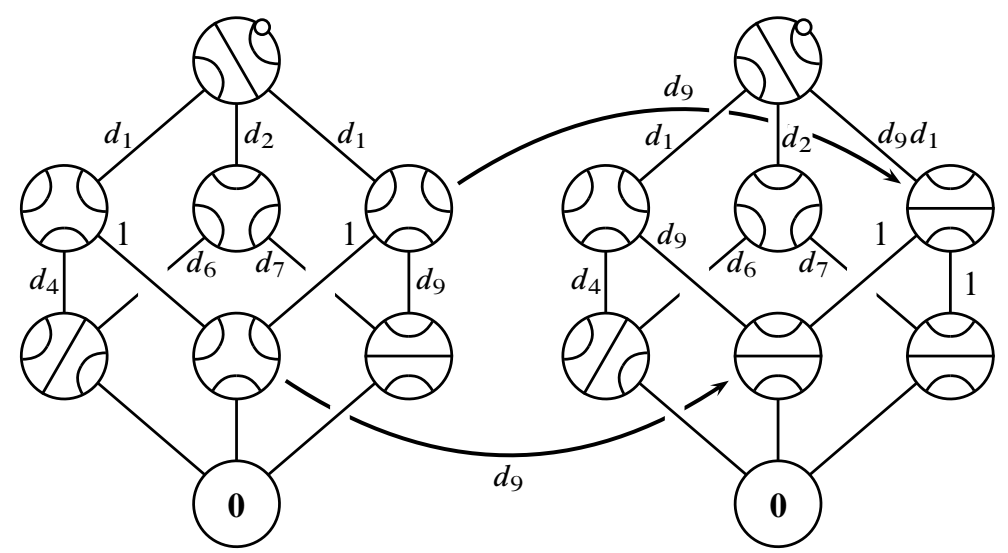

Now, $\sigma$ is neither injective or surjective so we turn to the homotopy tool. Using the contractibility of many of the hofibres we have from Lemma 3.4 and Corollary 3.9 that

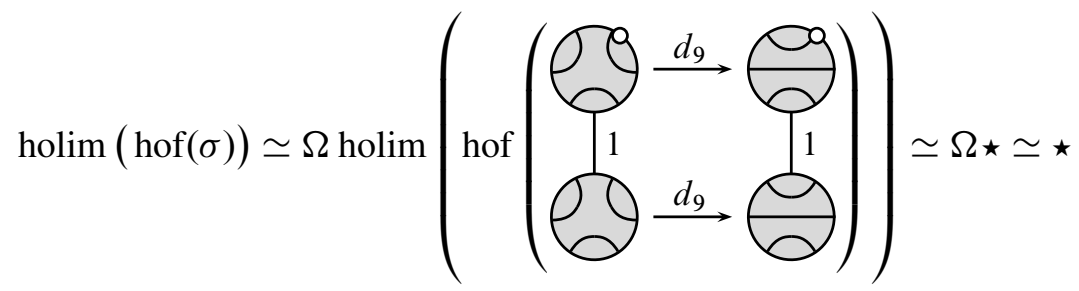

with the second to last homotopy equivalence from Corollary 3.7, Lemma 3.5 and Proposition 3.8. Thus by Proposition 3.2:

$$
\operatorname{hofibre}(\operatorname{holim} G \stackrel{\bar{\sigma}}{\rightarrow} \operatorname{holim}(H) \simeq \operatorname{holim} \operatorname{hof}(\sigma) \simeq \star
$$

It follows that $\bar{\sigma}$ is a homotopy equivalence from which we obtain

$$
\boldsymbol{Y}_{n} D=\operatorname{holim} F \simeq \operatorname{holim} G \simeq \operatorname{holim}(H) .
$$

Now repeat the entire process starting with $F_{K H}^{\prime}$, the Khovanov presheaf for $D^{\prime}$ : a short exact sequence $G_{0}^{\prime} \succ F_{K H}^{\prime} \rightarrow G^{\prime}$ and a morphism $\sigma^{\prime}: G^{\prime} \rightarrow H^{\prime}$ can be defined in a completely analogous way and the homotopy tool invoked to give

$$
\boldsymbol{Y}_{n} D^{\prime}=\operatorname{holim} F^{\prime} \simeq \operatorname{holim} G^{9} \simeq \operatorname{holim} H^{\prime} .
$$

Comparing $H$ and $H^{\prime}$, it turns out that the vertex groups are visibly identical, as are the edge maps except for an occurrence of $d_{9} d_{1}$ in $H$ and $d_{7} d_{2}$ in $H^{\prime}$. However the front top face of $F$ shows that $d_{9} d_{1}=d_{7} d_{2}$ and so $H$ and $H^{\prime}$ are in fact identical diagrams. Thus, $\boldsymbol{Y}_{n} D \simeq \boldsymbol{Y}_{n} D^{\prime}$, completing the proof of Reidemeister (III). 


\subsection{An example.}

We take the technology for a test drive by showing that

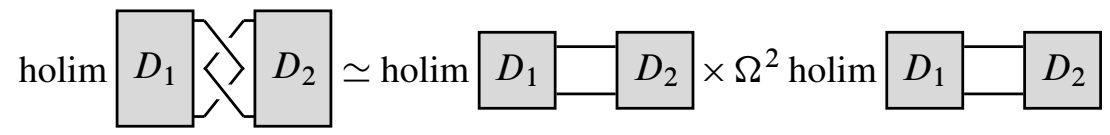

where $D_{1}, D_{2}$ are (unoriented) link diagrams and we are simplifying our pictograms as in the remarks immediately before Section 4.1. The conclusions for Khovanov homology are at the end of this calculation, and although they could be achieved at the level of chain complexes, our purpose here is to illustrate our machinery in action.

The Skein relation (Proposition 4.1) gives

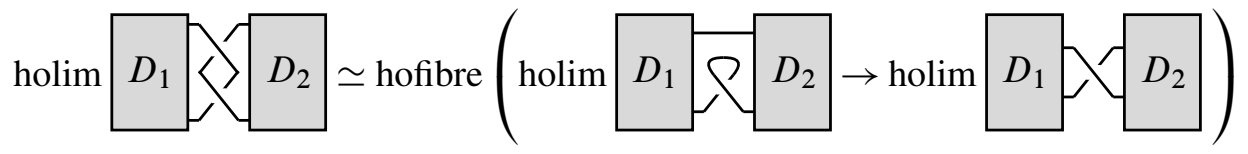

which in turn is homotopy equivalent to:

(†) holim holim
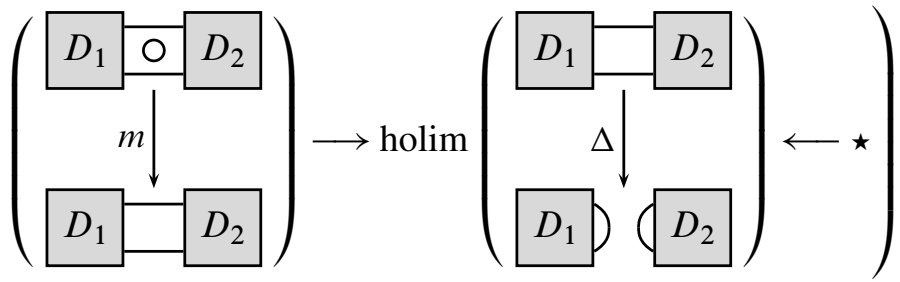

Consider the following two diagrams over $\boldsymbol{Q} \times \boldsymbol{D}$ (with $\boldsymbol{D}=\bullet \longrightarrow \bullet \longleftarrow \bullet$ ) and the morphism between them:

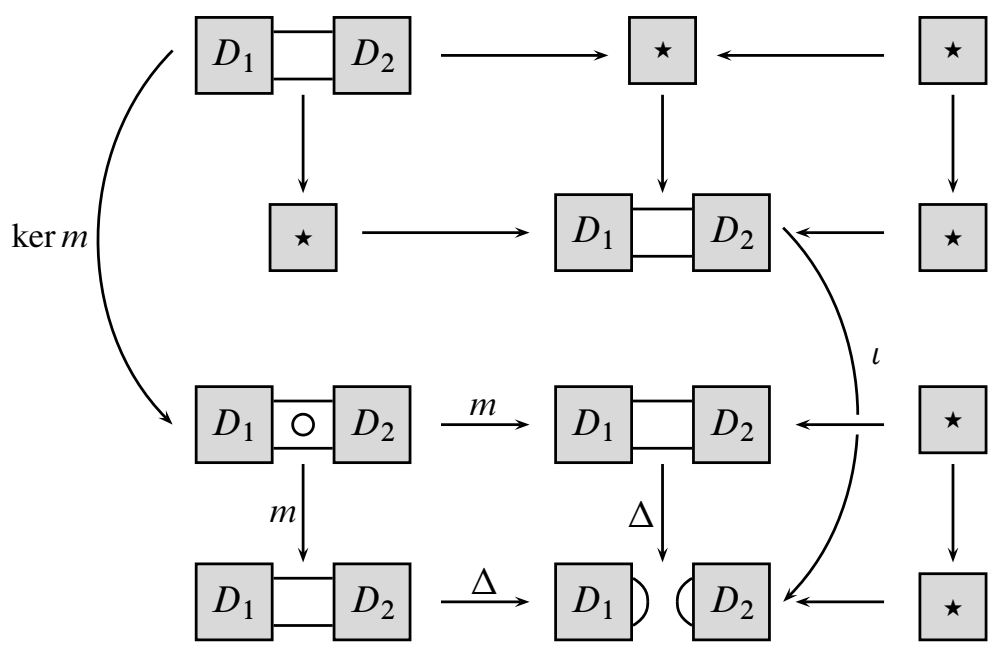


where $\star$ is the trivial diagram of spaces. We have the short exact sequence $H \succ F \rightarrow G$ of presheaves:

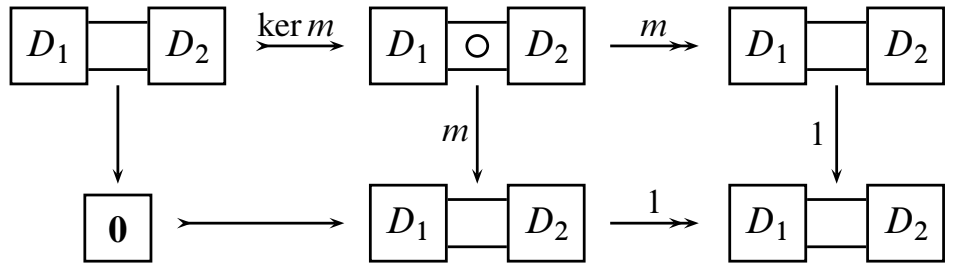

Here $\mathbf{0}$ the trivial presheaf, and $H^{\prime} \succ F^{\prime} \rightarrow G^{\prime}$ :

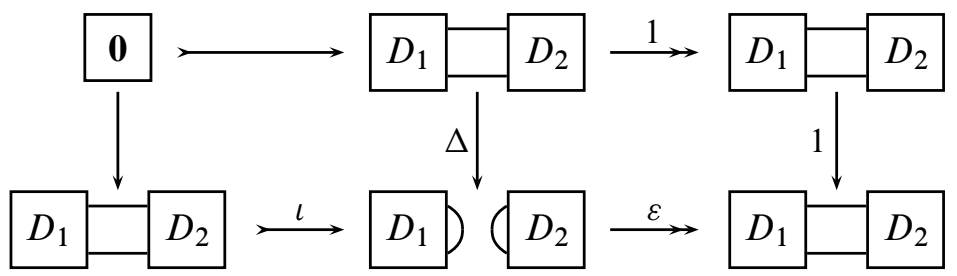

As $\mathscr{G}, \mathscr{G}^{\prime} \simeq \star$ by Lemma 3.5, the presheaf computational tool gives $\mathscr{H} \simeq \mathscr{F}, \mathscr{H}^{\prime} \simeq \mathscr{F}^{\prime}$. Proposition 2.1 applied to the morphism of diagrams over $\boldsymbol{Q} \times \boldsymbol{D}$ thus gives that $(\dagger)$ is homotopy equivalent to

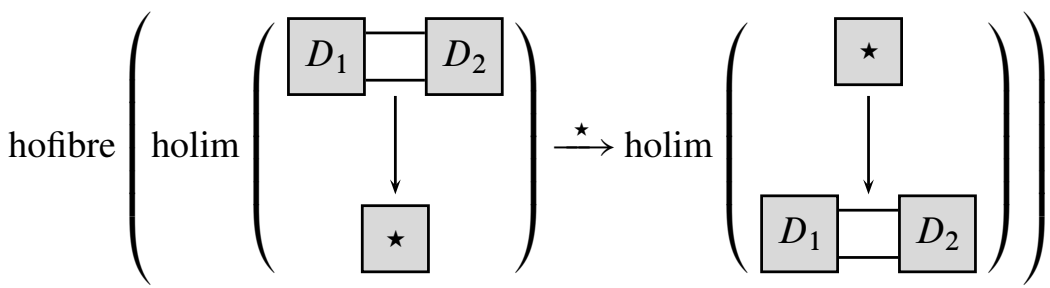

where $\stackrel{\star}{\longrightarrow}$ indicates that the induced map of the holim's factors through a point. The result then follows using hofibre $(X \stackrel{\star}{\longrightarrow} Y) \simeq X \times \Omega Y$, Lemma 3.3 and Corollary 3.9. To convert to a statement about Khovanov homology let $D_{1}, D_{2}$ now be oriented, with

$$
D_{1} \# \# D_{2}=D_{1} \lesssim D_{2} \text { and } D_{1} \# D_{2}=D_{1} \longrightarrow D_{2} \text {. }
$$

Then by the discussion at the end of Section 2.2

$$
\boldsymbol{Y}_{*}\left(D_{1} \# \# D_{2}\right) \simeq \boldsymbol{Y}_{*}\left(D_{1} \# D_{2}\right) \times \Omega^{2} \boldsymbol{Y}_{*}\left(D_{1} \# D_{2}\right)
$$

so that

$$
\boldsymbol{X}_{*}\left(D_{1} \# \# D_{2}\right) \simeq \boldsymbol{X}_{*}\left(D_{1} \# D_{2}\right) \times \Omega^{2} \boldsymbol{X}_{*}\left(D_{1} \# D_{2}\right)
$$


as the two additional crossings in $D_{1} \# \# D_{2}$ are both positive. Thus

$$
\begin{aligned}
K H^{i}\left(D_{1} \# \# D_{2}\right) & \cong \pi_{-i}\left(X_{*}\left(D_{1} \# D_{2}\right) \times \Omega^{2} X_{*}\left(D_{1} \# D_{2}\right)\right) \\
& \cong \pi_{-i} X_{*}\left(D_{1} \# D_{2}\right) \oplus \pi_{-i+2} X_{*}\left(D_{1} \# D_{2}\right) \\
& \cong K H^{i}\left(D_{1} \# D_{2}\right) \oplus K H^{i-2}\left(D_{1} \# D_{2}\right) .
\end{aligned}
$$

For example

$$
K H^{i} \bigcirc D D H^{i}(D) \oplus K H^{i-2}(D) .
$$

\section{References}

[1] D Bar-Natan, On Khovanov's categorification of the Jones polynomial, Algebr. Geom. Topol. 2 (2002) 337-370 MR1917056

[2] D Bar-Natan, Khovanov's homology for tangles and cobordisms, Geom. Topol. 9 (2005) 1443-1499 MR2174270

[3] A K Bousfield, D M Kan, Homotopy limits, completions and localizations, Lecture Notes in Mathematics 304, Springer, Berlin (1972) MR0365573

[4] E B Curtis, Simplicial homotopy theory, Advances in Math. 6 (1971) 107-209 MR0279808

[5] B Everitt, R Lipshitz, S Sarkar, P Turner, Khovanov homotopy types and the DoldThom functor arXiv:1202.1856

[6] B Everitt, P Turner, Homology of coloured posets: A generalisation of Khovanov's cube construction, J. Algebra 322 (2009) 429-448 MR2529096

[7] B Everitt, P Turner, Bundles of coloured posets and a Leray-Serre spectral sequence for Khovanov homology, Trans. Amer. Math. Soc. 364 (2012) 3137-3158 MR2888240

[8] T G Goodwillie, Calculus, II: Analytic functors, K-Theory 5 (1991/92) 295-332 MR1162445

[9] A Hatcher, Algebraic topology, Cambridge Univ. Press (2002) MR1867354

[10] M Khovanov, A categorification of the Jones polynomial, Duke Math. J. 101 (2000) 359-426 MR1740682

[11] M Khovanov, Link homology and categorification, from: "International Congress of Mathematicians, Vol. 2”, (M Sanz-Solé, J Soria, J L Varona, J Verdera, editors), Eur. Math. Soc., Zürich (2006) 989-999 MR2275632

[12] R Lipshitz, S Sarkar, A Khovanov homotopy type arXiv:1112.3932

[13] W S Massey, A basic course in algebraic topology, Graduate Texts in Mathematics 127, Springer, New York (1991) MR1095046 
[14] J P May, Simplicial objects in algebraic topology, Van Nostrand Math. Studies 11, Van Nostrand, Princeton, NJ (1967) MR0222892

[15] B Mitchell, Rings with several objects, Advances in Mathematics 8 (1972) 1-161 MR0294454

[16] I Moerdijk, Classifying spaces and classifying topoi, Lecture Notes in Mathematics 1616, Springer, Berlin (1995) MR1440857

[17] P Turner, Five lectures on Khovanov homology arXiv:math/0606464

[18] O Viro, Khovanov homology, its definitions and ramifications, Fund. Math. 184 (2004) 317-342 MR2128056

[19] C A Weibel, An introduction to homological algebra, Cambridge Studies Adv. Math. 38, Cambridge Univ. Press (1994) MR1269324

Department of Mathematics, University of York York YO10 5DD, UK

Section de Mathématiques, Université de Genève 2-4 rue du Lièvre, CH-1211 Geneva, Switzerland brent.everitt@york.ac.uk, prt.maths@gmail.com

Received: 2 July 2013 Revised: 5 December 2013 
